Article

\title{
Approximation Results for Equilibrium Problems Involving Strongly Pseudomonotone Bifunction in Real Hilbert Spaces
}

\author{
Wiyada Kumam ${ }^{1}$ and Kanikar Muangchoo ${ }^{2, *(1)}$ \\ 1 Program in Applied Statistics, Department of Mathematics and Computer Science, Faculty of Science and \\ Technology, Rajamangala University of Technology Thanyaburi, Thanyaburi, Pathumthani 12110, Thailand; \\ wiyada.kum@rmutt.ac.th \\ 2 Faculty of Science and Technology, Rajamangala University of Technology Phra Nakhon (RMUTP), \\ 1381 Pracharat 1 Road, Wongsawang, Bang Sue, Bangkok 10800, Thailand \\ * Correspondence: kanikar.m@rmutp.ac.th; Tel.: +66-0-2836-3000 (ext. 4193)
}

Received: 26 September 2020; Accepted: 21 November 2020; Published: 26 November 2020

check for updates

\begin{abstract}
A plethora of applications in non-linear analysis, including minimax problems, mathematical programming, the fixed-point problems, saddle-point problems, penalization and complementary problems, may be framed as a problem of equilibrium. Most of the methods used to solve equilibrium problems involve iterative methods, which is why the aim of this article is to establish a new iterative method by incorporating an inertial term with a subgradient extragradient method to solve the problem of equilibrium, which includes a bifunction that is strongly pseudomonotone and meets the Lipschitz-type condition in a real Hilbert space. Under certain mild conditions, a strong convergence theorem is proved, and a required sequence is generated without the information of the Lipschitz-type cost bifunction constants. Thus, the method operates with the help of a slow-converging step size sequence. In numerical analysis, we consider various equilibrium test problems to validate our proposed results.
\end{abstract}

Keywords: equilibrium problem; variational inequalities; strongly pseudomonotone bifunction; Lipschitz-type conditions

\section{Background}

Assume that a bifunction $f: \mathcal{H} \times \mathcal{H} \rightarrow \mathbb{R}$ satisfying the conditions $f(v, v)=0$ for each $v \in \mathcal{K}$. A equilibrium problem [1,2] for $f$ on $\mathcal{K}$ is said to be:

$$
\text { Find } v^{*} \in \mathcal{K} \text { such that } f\left(v^{*}, v\right) \geq 0, \forall v \in \mathcal{K} \text {. }
$$

where $\mathcal{K}$ is a non-empty closed and convex subset of a Hilbert space $\mathcal{H}$. Next, we present the definitions of the important classification of the problems of equilibrium [1,3]. A function $f: \mathcal{H} \times \mathcal{H} \rightarrow \mathbb{R}$ on $\mathcal{K}$ for $\gamma>0$ is said to be

(i) strongly monotone if

$$
f\left(v_{1}, v_{2}\right)+f\left(v_{2}, v_{1}\right) \leq-\gamma\left\|v_{1}-v_{2}\right\|^{2}, \forall v_{1}, v_{2} \in \mathcal{K}
$$

(ii) monotone if

$$
f\left(v_{1}, v_{2}\right)+f\left(v_{2}, v_{1}\right) \leq 0, \forall v_{1}, v_{2} \in \mathcal{K} ;
$$


(iii) $\gamma$-strongly pseudo-monotone if

$$
f\left(v_{1}, v_{2}\right) \geq 0 \Longrightarrow f\left(v_{2}, v_{1}\right) \leq-\gamma\left\|v_{1}-v_{2}\right\|^{2}, \forall v_{1}, v_{2} \in \mathcal{K} ;
$$

(iv) pseudo-monotone if

$$
f\left(v_{1}, v_{2}\right) \geq 0 \Longrightarrow f\left(v_{2}, v_{1}\right) \leq 0, \forall v_{1}, v_{2} \in \mathcal{K}
$$

and

(v) satisfy the Lipschitz-type conditions on $\mathcal{K}$ for $L_{1}, L_{2}>0$, such that

$$
f\left(v_{1}, v_{3}\right)-L_{1}\left\|v_{1}-v_{2}\right\|^{2}-L_{2}\left\|v_{2}-v_{3}\right\|^{2} \leq f\left(v_{1}, v_{2}\right)+f\left(v_{2}, v_{3}\right), \forall v_{1}, v_{2}, v_{3} \in \mathcal{K} .
$$

The above well-defined simple mathematical problem (1) includes many mathematical and applied sciences problems as a special case, consisting of the fixed point problems, vector and scalar minimization problems, problems of variational inequalities (VIP), the complementarity problems, the Nash equilibrium problems in non-cooperative games, and inverse optimization problems $[1,4,5]$. This problem is also seen as a problem of Ky Fan inequality based on his initial contribution [2]. Several researchers have developed and generalized numerous findings on the nature of a solution to an equilibrium problem. (e.g., see [2,4,6,7]). Due to the basic formulation of a problem (1) and its application in both the theoretical and applied sciences, it has been extensively studied in recent times by several authors $[8,9]$ (see also [10-16]).

Many methods have been previously established and considered their convergence investigation to deal with the problem (1). There is an impressive number of numerical methods have been designed along with their well-defined convergence analysis and theoretical properties to solve the problem (1) in different dimensional spaces [17-22]. Regularization is one of the most significant methods to figure out various ill-posed problems in the many fields of pure and applied mathematics. The prominent aspect of the regularization method is to employ it on monotone equilibrium problems and the initial problem converts into strongly monotone equilibrium sub-problem. Therefore, each computationally efficient sub-problem is strongly monotone and a unique solution exists.

A proximal method is another approach to deal with equilibrium problems that rely on numerical minimization problems [23]. This method has also been identified as the extragradient method [24] based on the initial contribution of the Korpelevich [25] method to solve the saddle point problems. Hieu [26] established an algorithmic sequence $\left\{u_{n}\right\}$ as follows:

$$
\left\{\begin{array}{l}
u_{0} \in \mathcal{K} \\
v_{n}=\underset{v \in \mathcal{K}}{\arg \min }\left\{\zeta_{n} f\left(u_{n}, v\right)+\frac{1}{2}\left\|u_{n}-v\right\|^{2}\right\} \\
u_{n+1}=\underset{v \in \mathcal{K}}{\arg \min }\left\{\zeta_{n} f\left(v_{n}, v\right)+\frac{1}{2}\left\|u_{n}-v\right\|^{2}\right\}
\end{array}\right.
$$

while $\left\{\zeta_{n}\right\}$ meet the following conditions:

$$
\mathcal{C}_{1}: \lim _{n \rightarrow+\infty} \zeta_{n}=0 \text { and } \mathcal{C}_{2}: \quad \sum_{n=1}^{+\infty} \zeta_{n}=+\infty
$$

Inertial-like methods are two-step iterative methods, where the next iteration is carried out by employing the previous two iterations $[27,28]$. The inertial interpolation term is required to boost the sequence and help to improve the convergence rate of the iterative sequence. Such inertial methods are essentially used to speed up the iterative sequence to the appropriate solution and to improve the convergence rate. Numerical descriptions demonstrate that inertial effects also enhance the numerical performance. Such impressive attributes increase the curiosity of researchers in creating inertial methods. Recently, various inertial methods have also been established for specific types of equilibrium problems [29-32]. 
In this paper, we use the projection method that is simple to carry out due to its low cost and efficient numerical computations. Inspired by the works of Fan et al. [33], Thong and Hieu [34], and Censor et al. [35], we set up an accelerated extragradient-like algorithm to solve the problem (1) and other special class of equilibrium problem, such as variational inequalities. We prove a strong convergence theorem corresponding to the sequence generated to solve the problem of equilibrium under certain mild conditions. At the end, the computational tests show that the algorithm is more efficient than the current ones [26,29,36-38].

The rest of the article has been organized as follows. Section 2 consists of some basic results which are used throughout the article. Section 3 includes our proposed method and its convergence analysis. Section 4 includes numerical experiments that demonstrate practical effectiveness.

\section{Preliminaries}

Assume that a convex function $g: \mathcal{K} \rightarrow \mathbb{R}$ and subdifferential of $g$ on $v_{1} \in \mathcal{K}$ is defined as follows:

$$
\partial g\left(v_{1}\right)=\left\{v_{3} \in \mathcal{H}: g\left(v_{2}\right)-g\left(v_{1}\right) \geq\left\langle v_{3}, v_{2}-v_{1}\right\rangle, \forall v_{2} \in \mathcal{K}\right\}
$$

A normal cone for $\mathcal{K}$ on $v_{1} \in \mathcal{K}$ is defined as follows:

$$
N_{\mathcal{K}}\left(v_{1}\right)=\left\{v_{3} \in \mathcal{H}:\left\langle v_{3}, v_{2}-v_{1}\right\rangle \leq 0, \forall v_{2} \in \mathcal{K}\right\}
$$

Lemma 1 ([39]). Assume the three sequences $\alpha_{n}, \beta_{n}$ and $\gamma_{n}$ are in $[0,+\infty)$ such that

$$
\alpha_{n+1} \leq \alpha_{n}+\beta_{n}\left(\alpha_{n}-\alpha_{n-1}\right)+\gamma_{n}, \text { for alln } \geq 1, \text { having } \sum_{n=1}^{+\infty} \gamma_{n}<+\infty,
$$

where $0<\beta$ with $0 \leq \beta_{n} \leq \beta<1$ for each $n \in \mathbb{N}$. Thus, we have

(i) $\sum_{n=1}^{+\infty}\left[\alpha_{n}-\alpha_{n-1}\right]_{+}<+\infty$, with $[q]_{+}:=\max \{q, 0\}$;

(ii) $\lim _{n \rightarrow+\infty} \alpha_{n}=\alpha^{*} \in[0,+\infty)$.

Lemma 2 ([40]). For each $v_{1}, v_{2} \in \mathcal{H}$ and $r \in \mathbb{R}$, the following equality holds

$$
\left\|r v_{1}+(1-r) v_{2}\right\|^{2}=r\left\|v_{1}\right\|^{2}+(1-r)\left\|v_{2}\right\|^{2}-r(1-r)\left\|v_{1}-v_{2}\right\|^{2} .
$$

Lemma 3 ([41]). Let $\left\{p_{n}\right\}$ and $\left\{q_{n}\right\} \subset[0,+\infty)$ be two sequences such that

$$
\sum_{n=1}^{+\infty} p_{n}=+\infty \quad \text { and } \quad \sum_{n=1}^{+\infty} p_{n} q_{n}<+\infty
$$

Then, $\liminf \operatorname{int}_{n \rightarrow+\infty} q_{n}=0$.

Lemma 4 ([42]). Assume that a function $h: \mathcal{K} \rightarrow \mathbb{R}$ is subdifferentiable, convex, and lower semi-continuous on $\mathcal{K}$. Then, $v_{1} \in \mathcal{K}$ is a function $h$ minimizer if and only if $0 \in \partial h\left(v_{1}\right)+N_{\mathcal{K}}\left(v_{1}\right)$ while $\partial h\left(v_{1}\right)$ and $N_{\mathcal{K}}\left(v_{1}\right)$ stand for the subdifferential of $h$ on $v_{1} \in \mathcal{K}$ and a normal cone of $\mathcal{K}$ at $v_{1}$, respectively.

Suppose that $f: \mathcal{H} \times \mathcal{H} \rightarrow \mathbb{R}$ satisfies the following conditions:

(C1) $f\left(v_{1}, v_{1}\right)=0$, for all $v_{1} \in \mathcal{K}$ and $f$ is strongly pseudomonotone on $\mathcal{K}$;

(C2) $f$ meet the Lipschitz-type condition with two constants $L_{1}$ and $L_{2}$; and

(C3) $f\left(v_{1},.\right)$ is convex and sub-differentiable on $\mathcal{H}$ for fixed each $v_{1} \in \mathcal{H}$. 


\section{Main Results}

The following is the main method (Algorithm 1) in more detail.

Algorithm 1. Modified subgradient extragradient method for equilibrium problems.

Step 0: Choose $u_{-1}, u_{0} \in \mathcal{H}$ arbitrarily. Let $\zeta_{n}$ satisfy the conditions (3). $\left\{\theta_{n}\right\}$ and $\left\{\vartheta_{n}\right\}$ are control parameter sequences.

Step 1: Compute

$$
v_{n}=\underset{v \in \mathcal{K}}{\arg \min }\left\{\zeta_{n} f\left(w_{n}, v\right)+\frac{1}{2}\left\|w_{n}-v\right\|^{2}\right\},
$$

where $w_{n}=u_{n}+\theta_{n}\left(u_{n}-u_{n-1}\right)$. If $v_{n}=w_{n}$, then STOP and $w_{n} \in E P(f, \mathcal{K})$.

Step 2: Compute a set

$$
\mathcal{H}_{n}=\left\{z \in \mathcal{H}:\left\langle w_{n}-\zeta_{n} t_{n}-v_{n}, z-v_{n}\right\rangle \leq 0\right\},
$$

where $t_{n} \in \partial_{2} f\left(w_{n}, v_{n}\right)$.

Step 3: Compute

Step 4: Compute

$$
\eta_{n}=\underset{v \in \mathcal{H}_{n}}{\arg \min }\left\{\zeta_{n} f\left(v_{n}, v\right)+\frac{1}{2}\left\|w_{n}-v\right\|^{2}\right\}
$$

$$
u_{n+1}=\left(1-\vartheta_{n}\right) w_{n}+\vartheta_{n} \eta_{n},
$$

where $\left\{\vartheta_{n}\right\}$ and $\left\{\theta_{n}\right\}$ are real sequences meet the conditions:

(i) $\left\{\theta_{n}\right\}$ sequence is non-decreasing and $0 \leq \theta_{n} \leq \theta<1$ for each $n \geq 1$;

(ii) there exists $\vartheta, \delta, \sigma>0$ such that

$$
\delta>\frac{4 \theta[\theta(1+\theta)+\sigma]}{1-\theta^{2}}
$$

and

$$
0<\vartheta \leq \vartheta_{n} \leq \frac{\delta-4 \theta\left[\theta(1+\theta)+\sigma+\frac{1}{4} \theta \delta\right]}{4 \delta\left[\theta(1+\theta)+\sigma+\frac{1}{4} \theta \delta\right]} .
$$

Set $n:=n+1$ and switch to Step 1 .

Lemma 5. Suppose that $f: \mathcal{H} \times \mathcal{H} \rightarrow \mathbb{R}$ satisfies the conditions (C1)-(C3). For $v^{*} \in E P(f, \mathcal{K}) \neq \varnothing$, we have

$$
\begin{aligned}
\left\|\eta_{n}-v^{*}\right\|^{2} \leq & \left\|w_{n}-v^{*}\right\|^{2}-\left(1-2 L_{1} \zeta_{n}\right)\left\|w_{n}-v_{n}\right\|^{2}-\left(1-2 L_{2} \zeta_{n}\right)\left\|\eta_{n}-v_{n}\right\|^{2} \\
& -2 \gamma \zeta_{n}\left\|v_{n}-v^{*}\right\|^{2} .
\end{aligned}
$$

Proof. By value of $\eta_{n}$ and Lemma 4 , we have

$$
0 \in \partial_{2}\left\{\zeta_{n} f\left(v_{n}, v\right)+\frac{1}{2}\left\|w_{n}-v\right\|^{2}\right\}\left(\eta_{n}\right)+N_{\mathcal{H}_{n}}\left(\eta_{n}\right) .
$$

Thus, there exists $\omega \in \partial f\left(v_{n}, \eta_{n}\right)$ and $\bar{\omega} \in N_{\mathcal{H}_{n}}\left(\eta_{n}\right)$ such that

$$
\zeta_{n} \omega+\eta_{n}-w_{n}+\bar{\omega}=0 .
$$

Thus, the above implies that

$$
\left\langle w_{n}-\eta_{n}, v-\eta_{n}\right\rangle=\zeta_{n}\left\langle\omega, v-\eta_{n}\right\rangle+\left\langle\bar{\omega}, v-\eta_{n}\right\rangle, \forall v \in \mathcal{H}_{n} .
$$


Since $\bar{\omega} \in N_{\mathcal{H}_{n}}\left(\eta_{n}\right)$, it implies that $\left\langle\bar{\omega}, v-\eta_{n}\right\rangle \leq 0$, for all $v \in \mathcal{H}_{n}$. This gives that

$$
\zeta_{n}\left\langle\omega, v-\eta_{n}\right\rangle \geq\left\langle w_{n}-\eta_{n}, v-\eta_{n}\right\rangle, \forall v \in \mathcal{H}_{n} .
$$

By $\omega \in \partial f\left(v_{n}, \eta_{n}\right)$, we have

$$
f\left(v_{n}, v\right)-f\left(v_{n}, \eta_{n}\right) \geq\left\langle\omega, v-\eta_{n}\right\rangle, \forall v \in \mathcal{H} .
$$

From (6) and (7), we obtain

$$
\zeta_{n} f\left(v_{n}, v\right)-\zeta_{n} f\left(v_{n}, \eta_{n}\right) \geq\left\langle w_{n}-\eta_{n}, v-\eta_{n}\right\rangle, \forall v \in \mathcal{H}_{n} .
$$

By the use of $v=v^{*}$, we get

$$
\zeta_{n} f\left(v_{n}, v^{*}\right)-\zeta_{n} f\left(v_{n}, \eta_{n}\right) \geq\left\langle w_{n}-\eta_{n}, v^{*}-\eta_{n}\right\rangle .
$$

By given $v^{*} \in E P(f, \mathcal{K}), f\left(v^{*}, v_{n}\right) \geq 0$, which implies that $f\left(v_{n}, v^{*}\right) \leq-\gamma\left\|v_{n}-v^{*}\right\|^{2}$. From the expression (9), we obtain

$$
\left\langle w_{n}-\eta_{n}, \eta_{n}-v^{*}\right\rangle \geq \zeta_{n} f\left(v_{n}, \eta_{n}\right)+\gamma \zeta_{n}\left\|v_{n}-v^{*}\right\|^{2} .
$$

Due to the Lipschitz-type continuity of a bifunction $f$,

$$
f\left(w_{n}, \eta_{n}\right) \leq f\left(w_{n}, v_{n}\right)+f\left(v_{n}, \eta_{n}\right)+L_{1}\left\|w_{n}-v_{n}\right\|^{2}+L_{2}\left\|v_{n}-\eta_{n}\right\|^{2} .
$$

Expressions (10) and (11) gives that

$$
\begin{aligned}
\left\langle w_{n}-\eta_{n}, \eta_{n}-v^{*}\right\rangle \geq & \zeta_{n}\left\{f\left(w_{n}, \eta_{n}\right)-f\left(w_{n}, v_{n}\right)\right\} \\
& -L_{1} \zeta_{n}\left\|w_{n}-v_{n}\right\|^{2}-L_{2} \zeta_{n}\left\|v_{n}-\eta_{n}\right\|^{2}+\gamma \zeta_{n}\left\|v_{n}-v^{*}\right\|^{2} .
\end{aligned}
$$

By value $\eta_{n} \in \mathcal{H}_{n}$,

$$
\left\langle w_{n}-\zeta_{n} t_{n}-v_{n}, \eta_{n}-v_{n}\right\rangle \leq 0
$$

The above implies that

$$
\left\langle w_{n}-v_{n}, \eta_{n}-v_{n}\right\rangle \leq \zeta_{n}\left\langle t_{n}, \eta_{n}-v_{n}\right\rangle .
$$

$t_{n} \in \partial_{2} f\left(w_{n}, v_{n}\right)$ gives that

$$
f\left(w_{n}, v\right)-f\left(w_{n}, v_{n}\right) \geq\left\langle t_{n}, v-v_{n}\right\rangle, \forall v \in \mathcal{H} .
$$

Substituting $v=\eta_{n}$ into the above expression,

$$
f\left(w_{n}, \eta_{n}\right)-f\left(w_{n}, v_{n}\right) \geq\left\langle t_{n}, \eta_{n}-v_{n}\right\rangle .
$$

Expressions (13) and (14) imply that

$$
\zeta_{n}\left\{f\left(w_{n}, \eta_{n}\right)-f\left(w_{n}, v_{n}\right)\right\} \geq\left\langle w_{n}-v_{n}, \eta_{n}-v_{n}\right\rangle .
$$

Combining expressions (12) and (15) implies that

$$
\begin{aligned}
\left\langle w_{n}-\eta_{n}, \eta_{n}-v^{*}\right\rangle \geq & \left\langle w_{n}-v_{n}, \eta_{n}-v_{n}\right\rangle \\
& -L_{1} \zeta_{n}\left\|w_{n}-v_{n}\right\|^{2}-L_{2} \zeta_{n}\left\|v_{n}-\eta_{n}\right\|^{2}+\gamma \zeta_{n}\left\|v_{n}-v^{*}\right\|^{2} .
\end{aligned}
$$


We have the following facts:

$$
\begin{aligned}
& 2\left\langle w_{n}-\eta_{n}, \eta_{n}-v^{*}\right\rangle=\left\|w_{n}-v^{*}\right\|^{2}-\left\|\eta_{n}-w_{n}\right\|^{2}-\left\|\eta_{n}-v^{*}\right\|^{2} . \\
& 2\left\langle v_{n}-w_{n}, v_{n}-\eta_{n}\right\rangle=\left\|w_{n}-v_{n}\right\|^{2}+\left\|\eta_{n}-v_{n}\right\|^{2}-\left\|w_{n}-\eta_{n}\right\|^{2} .
\end{aligned}
$$

Thus, we finally obtain

$$
\begin{aligned}
\left\|\eta_{n}-v^{*}\right\|^{2} \leq & \left\|w_{n}-v^{*}\right\|^{2}-\left(1-2 L_{1} \zeta_{n}\right)\left\|w_{n}-v_{n}\right\|^{2}-\left(1-2 L_{2} \zeta_{n}\right)\left\|\eta_{n}-v_{n}\right\|^{2} \\
& -2 \gamma \zeta_{n}\left\|v_{n}-v^{*}\right\|^{2}
\end{aligned}
$$

Theorem 1. The sequences $\left\{w_{n}\right\},\left\{v_{n}\right\},\left\{\eta_{n}\right\}$ and $\left\{u_{n}\right\}$ generated by Algorithm 1 strongly converge to $v^{*}$.

Proof. By the value of $u_{n+1}$, we have

$$
\begin{aligned}
\left\|u_{n+1}-v^{*}\right\|^{2} & =\left\|\left(1-\vartheta_{n}\right) w_{n}+\vartheta_{n} \eta_{n}-v^{*}\right\|^{2} \\
& =\left\|\left(1-\vartheta_{n}\right)\left(w_{n}-v^{*}\right)+\vartheta_{n}\left(\eta_{n}-v^{*}\right)\right\|^{2} \\
& =\left(1-\vartheta_{n}\right)\left\|w_{n}-v^{*}\right\|^{2}+\vartheta_{n}\left\|\eta_{n}-v^{*}\right\|^{2}-\vartheta_{n}\left(1-\vartheta_{n}\right)\left\|w_{n}-\eta_{n}\right\|^{2} \\
& \leq\left(1-\vartheta_{n}\right)\left\|w_{n}-v^{*}\right\|^{2}+\vartheta_{n}\left\|\eta_{n}-v^{*}\right\|^{2} .
\end{aligned}
$$

From Lemma 5, we obtain

$$
\begin{aligned}
\left\|\eta_{n}-v^{*}\right\|^{2} \leq & \left\|w_{n}-v^{*}\right\|^{2}-\left(1-2 L_{1} \zeta_{n}\right)\left\|w_{n}-v_{n}\right\|^{2}-\left(1-2 L_{2} \zeta_{n}\right)\left\|\eta_{n}-v_{n}\right\|^{2} \\
& -2 \gamma \zeta_{n}\left\|v_{n}-v^{*}\right\|^{2}
\end{aligned}
$$

By combining expressions (17) and (18), we get

$$
\begin{aligned}
\left\|u_{n+1}-v^{*}\right\|^{2} \leq & \left(1-\vartheta_{n}\right)\left\|w_{n}-v^{*}\right\|^{2}+\vartheta_{n}\left\|w_{n}-v^{*}\right\|^{2}-2 \gamma \vartheta_{n} \zeta_{n}\left\|v_{n}-v^{*}\right\|^{2} \\
& -\vartheta_{n}\left(1-2 L_{1} \zeta_{n}\right)\left\|w_{n}-v_{n}\right\|^{2}-\vartheta_{n}\left(1-2 L_{2} \zeta_{n}\right)\left\|\eta_{n}-v_{n}\right\|^{2} \\
= & \left\|w_{n}-v^{*}\right\|^{2}-\vartheta_{n}\left(1-b \zeta_{n}\right)\left[\left\|w_{n}-v_{n}\right\|^{2}+\left\|\eta_{n}-v_{n}\right\|^{2}\right] \\
= & \left\|w_{n}-v^{*}\right\|^{2}-\frac{\vartheta_{n}\left(1-b \zeta_{n}\right)}{2}\left[2\left\|w_{n}-v_{n}\right\|^{2}+2\left\|\eta_{n}-v_{n}\right\|^{2}\right] \\
\leq & \left\|w_{n}-v^{*}\right\|^{2}-\frac{\vartheta_{n}\left(1-b \zeta_{n}\right)}{2}\left[\left\|w_{n}-v_{n}\right\|+\left\|\eta_{n}-v_{n}\right\|\right]^{2} \\
\leq & \left\|w_{n}-v^{*}\right\|^{2}-\frac{\vartheta_{n}\left(1-b \zeta_{n}\right)}{2}\left\|\eta_{n}-w_{n}\right\|^{2}
\end{aligned}
$$

where $b=\max \left\{2 L_{1}, 2 L_{2}\right\}$. It continues from $u_{n+1}$ such that

$$
\left\|u_{n+1}-w_{n}\right\|=\left\|\left(1-\vartheta_{n}\right) w_{n}+\vartheta_{n} \eta_{n}-w_{n}\right\|=\left\|\vartheta_{n}\left(\eta_{n}-w_{n}\right)\right\|
$$

Combining (21) and (22), we have

$$
\left\|u_{n+1}-v^{*}\right\|^{2} \leq\left\|w_{n}-v^{*}\right\|^{2}-\frac{\left(1-b \zeta_{n}\right)}{2 \vartheta_{n}}\left\|u_{n+1}-w_{n}\right\|^{2} .
$$

Since $\zeta_{n} \rightarrow 0$, thus there is $n_{0}>0$ in order that $\zeta_{n} \leq \frac{1}{2 b}$ for each $n \geq n_{0}$. This implies $\frac{1-b \zeta_{n}}{2} \geq \frac{1}{4}$ for every $n \geq n_{0}$. The expression (23) for $n \geq n_{0}$, turn as

$$
\left\|u_{n+1}-v^{*}\right\|^{2} \leq\left\|w_{n}-v^{*}\right\|^{2}-\frac{1}{4 \vartheta_{n}}\left\|u_{n+1}-w_{n}\right\|^{2} .
$$


By description of $w_{n}$, we have

$$
\begin{aligned}
\left\|w_{n}-v^{*}\right\|^{2} & =\left\|u_{n}+\theta_{n}\left(u_{n}-u_{n-1}\right)-v^{*}\right\|^{2} \\
& =\left\|\left(1+\theta_{n}\right)\left(u_{n}-v^{*}\right)-\theta_{n}\left(u_{n-1}-v^{*}\right)\right\|^{2} \\
& =\left(1+\theta_{n}\right)\left\|u_{n}-v^{*}\right\|^{2}-\theta_{n}\left\|u_{n-1}-v^{*}\right\|^{2}+\theta_{n}\left(1+\theta_{n}\right)\left\|u_{n}-u_{n-1}\right\|^{2} .
\end{aligned}
$$

By value of $w_{n}$, we have

$$
\begin{aligned}
\left\|u_{n+1}-w_{n}\right\|^{2} & =\left\|u_{n+1}-u_{n}-\theta_{n}\left(u_{n}-u_{n-1}\right)\right\|^{2} \\
& =\left\|u_{n+1}-u_{n}\right\|^{2}+\theta_{n}^{2}\left\|u_{n}-u_{n-1}\right\|^{2}+2 \theta_{n}\left\langle u_{n}-u_{n+1}, u_{n}-u_{n-1}\right\rangle \\
& \geq\left\|u_{n+1}-u_{n}\right\|^{2}+\theta_{n}^{2}\left\|u_{n}-u_{n-1}\right\|^{2}-\rho_{n} \theta_{n}\left\|u_{n+1}-u_{n}\right\|^{2}-\frac{\theta_{n}}{\rho_{n}}\left\|u_{n}-u_{n-1}\right\|^{2} \\
& \geq\left(1-\rho_{n} \theta_{n}\right)\left\|u_{n+1}-u_{n}\right\|^{2}+\left(\theta_{n}^{2}-\frac{\theta_{n}}{\rho_{n}}\right)\left\|u_{n}-u_{n-1}\right\|^{2}
\end{aligned}
$$

where $\rho_{n}=\frac{1}{\delta \vartheta_{n}+\theta_{n}}$. Combining (24), (25), and (27) gives that

$$
\begin{aligned}
\left\|u_{n+1}-v^{*}\right\|^{2} \leq & \left(1+\theta_{n}\right)\left\|u_{n}-v^{*}\right\|^{2}-\theta_{n}\left\|u_{n-1}-v^{*}\right\|^{2}+\theta_{n}\left(1+\theta_{n}\right)\left\|u_{n}-u_{n-1}\right\|^{2} \\
& -\frac{1}{4 \vartheta_{n}}\left[\left(1-\rho_{n} \theta_{n}\right)\left\|u_{n+1}-u_{n}\right\|^{2}+\left(\theta_{n}^{2}-\frac{\theta_{n}}{\rho_{n}}\right)\left\|u_{n}-u_{n-1}\right\|^{2}\right] \\
= & \left(1+\theta_{n}\right)\left\|u_{n}-v^{*}\right\|^{2}-\theta_{n}\left\|u_{n-1}-v^{*}\right\|^{2}-\frac{1}{4 \vartheta_{n}}\left(1-\rho_{n} \theta_{n}\right)\left\|u_{n+1}-u_{n}\right\|^{2} \\
& +\left[\theta_{n}\left(1+\theta_{n}\right)-\frac{1}{4 \vartheta_{n}}\left(\theta_{n}^{2}-\frac{\theta_{n}}{\rho_{n}}\right)\right]\left\|u_{n}-u_{n-1}\right\|^{2} \\
= & \left(1+\theta_{n}\right)\left\|u_{n}-v^{*}\right\|^{2}-\theta_{n}\left\|u_{n-1}-v^{*}\right\|^{2}-\frac{1}{4 \vartheta_{n}}\left(1-\rho_{n} \theta_{n}\right)\left\|u_{n+1}-u_{n}\right\|^{2} \\
& +\gamma_{n}\left\|u_{n}-u_{n-1}\right\|^{2},
\end{aligned}
$$

where

$$
\gamma_{n}=\theta_{n}\left(1+\theta_{n}\right)-\frac{1}{4 \vartheta_{n}}\left(\theta_{n}^{2}-\frac{\theta_{n}}{\rho_{n}}\right)=\theta_{n}\left(1+\theta_{n}\right)+\frac{1}{4 \vartheta_{n}}\left(\frac{\theta_{n}}{\rho_{n}}-\theta_{n}^{2}\right)>0 .
$$

By the above expression and the choice of $\left\{\rho_{n}\right\}$, we have

$$
\gamma_{n}=\theta_{n}\left(1+\theta_{n}\right)+\frac{1}{4 \vartheta_{n}}\left(\frac{\theta_{n}}{\rho_{n}}-\theta_{n}^{2}\right) \leq \theta(1+\theta)+\frac{1}{4} \theta \delta .
$$

We substitute

$$
\Psi_{n}=\left\|u_{n}-p\right\|^{2}-\theta_{n}\left\|u_{n-1}-p\right\|^{2}+\gamma_{n}\left\|u_{n}-u_{n-1}\right\|^{2} .
$$

It follows (29) such that

$$
\begin{aligned}
\Psi_{n+1}-\Psi_{n}= & \left\|u_{n+1}-p\right\|^{2}-\theta_{n+1}\left\|u_{n}-p\right\|^{2}+\gamma_{n+1}\left\|u_{n+1}-u_{n}\right\|^{2} \\
& -\left\|u_{n}-p\right\|^{2}+\theta_{n}\left\|u_{n-1}-p\right\|^{2}-\gamma_{n}\left\|u_{n}-u_{n-1}\right\|^{2} \\
\leq & \left\|u_{n+1}-p\right\|^{2}-\left(1+\theta_{n}\right)\left\|u_{n}-p\right\|^{2}+\theta_{n}\left\|u_{n-1}-p\right\|^{2} \\
& +\gamma_{n+1}\left\|u_{n+1}-u_{n}\right\|^{2}-\gamma_{n}\left\|u_{n}-u_{n-1}\right\|^{2} \\
= & -\left(\frac{1}{4 \vartheta_{n}}\left(1-\rho_{n} \theta_{n}\right)-\gamma_{n+1}\right)\left\|u_{n+1}-u_{n}\right\|^{2} .
\end{aligned}
$$

We claim that

$$
\frac{1}{4 \vartheta_{n}}\left(1-\rho_{n} \theta_{n}\right)-\gamma_{n+1} \geq \sigma .
$$


The above inequality implies that

$$
\begin{aligned}
\frac{1}{4 \vartheta_{n}}\left(1-\rho_{n} \theta_{n}\right)-\gamma_{n+1} & \geq \sigma \\
\text { iff } \quad\left(1-\rho_{n} \theta_{n}\right)-4 \vartheta_{n} \gamma_{n+1} & \geq 4 \vartheta_{n} \sigma \\
\text { iff } \quad\left(1-\rho_{n} \theta_{n}\right)-4 \vartheta_{n}\left(\gamma_{n+1}+\sigma\right) & \geq 0 \\
\text { iff } \quad \frac{\delta \vartheta_{n}}{\delta \vartheta_{n}+\theta_{n}}-4 \vartheta_{n}\left(\gamma_{n+1}+\sigma\right) & \geq 0 \\
\text { iff } \quad-4\left(\gamma_{n+1}+\sigma\right)\left(\delta \vartheta_{n}+\theta_{n}\right) & \geq-\delta
\end{aligned}
$$

(31) and (5) give that

$$
-4\left(\gamma_{n+1}+\sigma\right)\left(\delta \vartheta_{n}+\theta_{n}\right) \geq-4\left[\theta(1+\theta)+\frac{1}{4} \theta \delta+\sigma\right]\left(\delta \vartheta_{n}+\theta_{n}\right) \geq-\delta .
$$

Expression (32) implies that

$$
\Psi_{n+1}-\Psi_{n} \leq-\sigma\left\|u_{n+1}-u_{n}\right\|^{2} \leq 0, \quad \text { for all } n \geq n_{0} .
$$

Thus, we obtain a non-increasing sequence $\left\{\Psi_{n}\right\}$ for $n \geq n_{0}$. By the value of $\Psi_{n+1}$, we have

$$
\begin{aligned}
\Psi_{n+1} & =\left\|u_{n+1}-p\right\|^{2}-\theta_{n+1}\left\|u_{n}-p\right\|^{2}+\gamma_{n+1}\left\|u_{n+1}-u_{n}\right\|^{2} \\
& \geq-\theta_{n+1}\left\|u_{n}-p\right\|^{2} .
\end{aligned}
$$

By the value of $\Psi_{n}$, we have

$$
\begin{aligned}
\Psi_{n} & =\left\|u_{n}-p\right\|^{2}-\theta_{n}\left\|u_{n-1}-p\right\|^{2}+\gamma_{n}\left\|u_{n}-u_{n-1}\right\|^{2} \\
& \geq\left\|u_{n}-p\right\|^{2}-\theta_{n}\left\|u_{n-1}-p\right\|^{2} .
\end{aligned}
$$

Thus, expression (37) for $n \geq n_{0}$ is such that

$$
\begin{aligned}
\left\|u_{n}-p\right\|^{2} & \leq \Psi_{n}+\theta_{n}\left\|u_{n-1}-p\right\|^{2} \\
& \leq \Psi_{n_{0}}+\theta\left\|u_{n-1}-p\right\|^{2} \\
& \leq \cdots \leq \Psi_{n_{0}}\left(\theta^{n-n_{0}}+\cdots+1\right)+\theta^{n-n_{0}}\left\|u_{n_{0}}-p\right\|^{2} \\
& \leq \frac{\Psi_{n_{0}}}{1-\theta}+\theta^{n-n_{0}}\left\|u_{n_{0}}-p\right\|^{2} .
\end{aligned}
$$

By (36) and (38) for all $n \geq n_{0}$, we get

$$
\begin{aligned}
-\Psi_{n+1} & \leq \theta_{n+1}\left\|u_{n}-p\right\|^{2} \\
& \leq \theta\left\|u_{n}-p\right\|^{2} \\
& \leq \theta \frac{\Psi_{n_{0}}}{1-\theta}+\theta^{n-n_{0}+1}\left\|u_{n_{0}}-p\right\|^{2} .
\end{aligned}
$$

It follows from (35) and (39) that

$$
\begin{aligned}
\sigma \sum_{n=n_{0}}^{k}\left\|u_{n+1}-u_{n}\right\|^{2} & \leq \Psi_{n_{0}}-\Psi_{k+1} \\
& \leq \Psi_{n_{0}}+\theta \frac{\Psi_{n_{0}}}{1-\theta}+\theta^{n-n_{0}+1}\left\|u_{n_{0}}-p\right\|^{2} \\
& \leq \frac{\Psi_{n_{0}}}{1-\theta}+\left\|u_{n_{0}}-p\right\|^{2} .
\end{aligned}
$$


Sending $k \rightarrow+\infty$ implies that

$$
\sum_{n=1}^{+\infty}\left\|u_{n+1}-u_{n}\right\|^{2}<+\infty
$$

It continues from that

$$
\lim _{n \rightarrow+\infty}\left\|u_{n+1}-u_{n}\right\|=0 .
$$

Equations (26) and (42) provide that

$$
\lim _{n \rightarrow+\infty}\left\|u_{n+1}-w_{n}\right\|=0 .
$$

By the value of $u_{n+1}$, we have

$$
\left\|u_{n+1}-w_{n}\right\|=\left\|\left(1-\vartheta_{n}\right) w_{n}+\vartheta_{n} \eta_{n}-w_{n}\right\|=\vartheta_{n}\left\|\eta_{n}-w_{n}\right\| .
$$

By Equations (43) and (44), we obtain

$$
\lim _{n \rightarrow+\infty}\left\|\eta_{n}-w_{n}\right\|=0
$$

By the use of triangular inequality and (42) with (43), we obtain

$$
\lim _{n \rightarrow+\infty}\left\|u_{n}-w_{n}\right\| \leq \lim _{n \rightarrow+\infty}\left\|u_{n}-u_{n+1}\right\|+\lim _{n \rightarrow+\infty}\left\|u_{n+1}-w_{n}\right\|=0
$$

and

$$
\lim _{n \rightarrow+\infty}\left\|u_{n}-\eta_{n}\right\| \leq \lim _{n \rightarrow+\infty}\left\|u_{n}-w_{n}\right\|+\lim _{n \rightarrow+\infty}\left\|w_{n}-\eta_{n}\right\|=0 .
$$

Expressions (28) and (41) with Lemma 1 imply that

$$
\lim _{n \rightarrow+\infty}\left\|u_{n}-v^{*}\right\|^{2}=b \quad \text { for some } \quad b \geq 0 .
$$

Expressions (46) and (47) imply that

$$
\lim _{n \rightarrow+\infty}\left\|w_{n}-v^{*}\right\|^{2}=\lim _{n \rightarrow+\infty}\left\|\eta_{n}-v^{*}\right\|^{2}=b .
$$

Thus, Lemma 5 implies that

$$
\left(1-2 L_{2} \zeta\right)\left\|w_{n}-v_{n}\right\|^{2} \leq\left\|w_{n}-v^{*}\right\|^{2}-\left\|\eta_{n}-v^{*}\right\|^{2} .
$$

The above expression with (48) and (49) gives that

$$
\lim _{n \rightarrow+\infty}\left\|w_{n}-v_{n}\right\|=0 \text { and } \lim _{n \rightarrow+\infty}\left\|v_{n}-v^{*}\right\|^{2}=b .
$$

The argument referred to above concludes that the sequences $\left\{w_{n}\right\},\left\{v_{n}\right\},\left\{\eta_{n}\right\}$, and $\left\{\eta_{n}\right\}$ are bounded for each $v^{*} \in E P(f, \mathcal{K})$ the $\lim _{n \rightarrow+\infty}\left\|u_{n}-v^{*}\right\|^{2}$ exists. It follows from (19) and (25) that we have

$$
\begin{aligned}
2 \gamma \vartheta_{n} \zeta_{n}\left\|v_{n}-v^{*}\right\|^{2} \leq & -\left\|u_{n+1}-v^{*}\right\|^{2}+\left(1+\theta_{n}\right)\left\|u_{n}-v^{*}\right\|^{2}-\theta_{n}\left\|u_{n-1}-v^{*}\right\|^{2} \\
& +\theta_{n}\left(1+\theta_{n}\right)\left\|u_{n}-u_{n-1}\right\|^{2} \\
\leq & \left(\left\|u_{n}-v^{*}\right\|^{2}-\left\|u_{n+1}-v^{*}\right\|^{2}\right)+2 \theta\left\|u_{n}-u_{n-1}\right\|^{2} \\
& +\left(\theta_{n}\left\|u_{n}-v^{*}\right\|^{2}-\theta_{n-1}\left\|u_{n-1}-v^{*}\right\|^{2}\right) .
\end{aligned}
$$


The above expression for $k \geq n_{0}$ gives that

$$
\begin{aligned}
\sum_{n=n_{0}}^{k} 2 \gamma \vartheta_{n} \zeta_{n}\left\|v_{n}-v^{*}\right\|^{2} \leq & \left(\left\|u_{n_{0}}-v^{*}\right\|^{2}-\left\|u_{k+1}-v^{*}\right\|^{2}\right)+2 \theta \sum_{n=n_{0}}^{k}\left\|u_{n}-u_{n-1}\right\|^{2} \\
& +\left(\theta_{k}\left\|u_{k}-v^{*}\right\|^{2}-\theta_{0}\left\|u_{n_{0}}-v^{*}\right\|^{2}\right) \\
\leq & \left\|u_{n_{0}}-v^{*}\right\|^{2}+\theta\left\|u_{k}-v^{*}\right\|^{2}+2 \theta \sum_{n=n_{0}}^{k}\left\|u_{n}-u_{n-1}\right\|^{2}
\end{aligned}
$$

letting $k \rightarrow+\infty$ in (53), we obtain

$$
\sum_{n=n_{0}}^{k} 2 \gamma \vartheta_{n} \zeta_{n}\left\|v_{n}-v^{*}\right\|^{2}<+\infty
$$

From Lemma 3 and (54),

$$
\liminf \left\|v_{n}-p\right\|=0 .
$$

By expressions (46), (47), (49), (51) and (55),

$$
\lim _{n \rightarrow+\infty}\left\|v_{n}-p\right\|=\lim _{n \rightarrow+\infty}\left\|w_{n}-p\right\|=\lim _{n \rightarrow+\infty}\left\|\eta_{n}-p\right\|=\lim _{n \rightarrow+\infty}\left\|u_{n}-p\right\|=0 .
$$

This completes the proof.

Next, we consider the application of our results to solve variational inequality problems. A function $G: \mathcal{H} \rightarrow \mathcal{H}$ is said to be

(G1) strongly pseudo-monotone over $\mathcal{K}$ for $\gamma>0$ if

$$
\left\langle G\left(v_{1}\right), v_{2}-v_{1}\right\rangle \geq 0 \quad \text { implies that }\left\langle G\left(v_{2}\right), v_{1}-v_{2}\right\rangle \leq-\gamma\left\|v_{1}-v_{2}\right\|^{2}, \forall v_{1}, v_{2} \in \mathcal{K} ;
$$

and

(G2) L-Lipschitz continuity on $C$ if

$$
\left\|G\left(v_{1}\right)-G\left(v_{2}\right)\right\| \leq L\left\|v_{1}-v_{2}\right\|, \forall v_{1}, v_{2} \in \mathcal{K} .
$$

Let a bifunction $f\left(v_{1}, v_{2}\right):=\left\langle G\left(v_{1}\right), v_{2}-v_{1}\right\rangle$ for all $v_{1}, v_{2} \in \mathcal{K}$ then equilibrium problem turns into problem of variational inequality with $L=2 L_{1}=2 L_{2}$. By the value of $v_{n}$,

$$
\begin{aligned}
v_{n} & =\underset{v \in \mathcal{K}}{\arg \min }\left\{\zeta_{n} f\left(w_{n}, v\right)+\frac{1}{2}\left\|w_{n}-v\right\|^{2}\right\} \\
& =\underset{v \in \mathcal{K}}{\arg \min }\left\{\zeta_{n}\left\langle G\left(w_{n}\right), v-w_{n}\right\rangle+\frac{1}{2}\left\|w_{n}-v\right\|^{2}\right\} \\
& =\underset{v \in \mathcal{K}}{\arg \min }\left\{\zeta_{n}\left\langle G\left(w_{n}\right), v-w_{n}\right\rangle+\frac{1}{2}\left\|w_{n}-v\right\|^{2}+\frac{\zeta_{n}^{2}}{2}\left\|G\left(w_{n}\right)\right\|^{2}-\frac{\zeta_{n}^{2}}{2}\left\|G\left(w_{n}\right)\right\|^{2}\right\} \\
& =\underset{v \in \mathcal{K}}{\arg \min }\left\{\frac{1}{2}\left\|v-\left(w_{n}-\zeta_{n} G\left(w_{n}\right) \|^{2}\right\}-\frac{\zeta_{n}^{2}}{2}\right\| G\left(w_{n}\right) \|^{2}\right. \\
& =P_{\mathcal{K}}\left(w_{n}-\zeta_{n} G\left(w_{n}\right)\right) .
\end{aligned}
$$

Similar to above, the value of $\eta_{n}$ turns into

$$
\eta_{n}=P_{\mathcal{H}_{n}}\left(w_{n}-\zeta_{n} G\left(v_{n}\right)\right) .
$$


Corollary 1. Assume that an operator $G: \mathcal{K} \rightarrow \mathcal{H}$ satisfies Conditions (G1)-(G2). Let $\left\{w_{n}\right\},\left\{v_{n}\right\},\left\{\eta_{n}\right\}$, and $\left\{u_{n}\right\}$ be the sequences generated as follows:

(S1) Let $u_{-1}, u_{0} \in \mathcal{H}$ arbitrarily.

(S2) Choose $\zeta_{n}$ satisfying condition (3) and $\left\{\theta_{n}\right\},\left\{\vartheta_{n}\right\}$ are control parameters.

(S3) Compute

$$
v_{n}=P_{\mathcal{K}}\left(w_{n}-\zeta_{n} G\left(w_{n}\right)\right),
$$

where $w_{n}=u_{n}+\theta_{n}\left(u_{n}-u_{n-1}\right)$. If $v_{n}=w_{n}$, then STOP.

(S4) Determine a half space first $\mathcal{H}_{n}=\left\{z \in \mathcal{H}:\left\langle w_{n}-\zeta_{n} G\left(w_{n}\right)-v_{n}, z-v_{n}\right\rangle \leq 0\right\}$ and evaluate

$$
\eta_{n}=P_{\mathcal{H}_{n}}\left(w_{n}-\zeta_{n} G\left(v_{n}\right)\right) .
$$

(S5) Compute

$$
u_{n+1}=\left(1-\vartheta_{n}\right) w_{n}+\vartheta_{n} \eta_{n}
$$

where $\left\{\theta_{n}\right\}$ and $\left\{\vartheta_{n}\right\}$ satisfies the following conditions:

(i) non-decreasing sequence $\left\{\theta_{n}\right\}$ through $0 \leq \theta_{n} \leq \theta<1$, for each $n \geq 1$; and

(ii) there exists $\vartheta, \delta, \sigma>0$, thus that

$$
\delta>\frac{4 \theta[\theta(1+\theta)+\sigma]}{1-\theta^{2}}
$$

and

$$
0<\vartheta \leq \vartheta_{n} \leq \frac{\delta-4 \theta\left[\theta(1+\theta)+\sigma+\frac{1}{4} \theta \delta\right]}{4 \delta\left[\theta(1+\theta)+\sigma+\frac{1}{4} \theta \delta\right]}
$$

Then, $\left\{w_{n}\right\},\left\{v_{n}\right\},\left\{\eta_{n}\right\}$, and $\left\{u_{n}\right\}$ strongly converge to $v^{*} \in V I(G, \mathcal{K})$.

\section{Numerical Illustration}

Numerical findings are summarized in this section to demonstrate the effectiveness of the proposed methods. The following control parameters are used in this section.

(1) For Hieu et al. [26] (Hieu-EgA), we use $D_{n}=\left\|u_{n}-v_{n}\right\|^{2}$.

(2) For Hieu et al. [29] (Hieu-mEgA), we use $\theta=0.5$ and $D_{n}=\max \left\{\left\|u_{n+1}-v_{n}\right\|^{2},\left\|u_{n+1}-w_{n}\right\|^{2}\right\}$.

(3) For Algorithm 1 (iEgA), we use $\alpha_{n}=0.50, \beta_{n}=0.80$, and $D_{n}=\left\|w_{n}-v_{n}\right\|^{2}$.

Example 1. Let bifunction $f$ have the following form

$$
f(u, v)=\langle A u+B v+c, v-u\rangle
$$

where $c \in \mathbb{R}^{5}$ and $A$ and $B$ are

$$
A=\left(\begin{array}{ccccc}
3.1 & 2 & 0 & 0 & 0 \\
2 & 3.6 & 0 & 0 & 0 \\
0 & 0 & 3.5 & 2 & 0 \\
0 & 0 & 2 & 3.3 & 0 \\
0 & 0 & 0 & 0 & 3
\end{array}\right) \quad B=\left(\begin{array}{ccccc}
1.6 & 1 & 0 & 0 & 0 \\
1 & 1.6 & 0 & 0 & 0 \\
0 & 0 & 1.5 & 1 & 0 \\
0 & 0 & 1 & 1.5 & 0 \\
0 & 0 & 0 & 0 & 2
\end{array}\right)
$$


and

$$
c=\left(\begin{array}{c}
1 \\
-2 \\
-1 \\
2 \\
-1
\end{array}\right)
$$

where Lipschitz parameters $L_{1}=L_{2}=\frac{1}{2}\|A-B\|[26]$. The feasible set $\mathcal{K} \subset \mathbb{R}^{5}$ is

$$
\mathcal{K}:=\left\{u \in \mathbb{R}^{5}:-5 \leq u_{i} \leq 5\right\} .
$$

Table 1 and Figures $1-3$ show the numerical results by $u_{-1}=u_{0}=v_{0}=(1, \cdots, 1)$, and TOL $=10^{-12}$.

\begin{tabular}{|c|c|c|c|c|c|c|c|c|}
\hline \multirow[b]{2}{*}{$\mathbf{n}$} & \multirow[b]{2}{*}{ TOL } & \multirow[b]{2}{*}{$\zeta_{n}$} & \multicolumn{2}{|c|}{ Hieu-EgA [26] } & \multicolumn{2}{|c|}{ Hieu-mEgA [29] } & \multirow{2}{*}{$\begin{array}{l}\text { iEgA } \\
\text { Iter. }\end{array}$} & \multirow{2}{*}{$\begin{array}{c}\text { Algorithm } 1 \\
\text { Time }\end{array}$} \\
\hline & & & Iter. & Time & Iter. & Time & & \\
\hline 5 & $10^{-12}$ & $\frac{1}{\log (n+3)(n+1)}$ & 320 & 5.8584 & 59 & 0.5979 & 64 & 0.2830 \\
\hline 5 & $10^{-12}$ & $\frac{1}{n+1}$ & 222 & 3.1116 & 43 & 0.4158 & 39 & 0.1696 \\
\hline 5 & $10^{-12}$ & $\frac{\log (n+3)}{n+1}$ & 122 & 1.5466 & 40 & 0.3732 & 33 & 0.1581 \\
\hline
\end{tabular}

Table 1. Example 1: Numerical values for Figures 1-3.

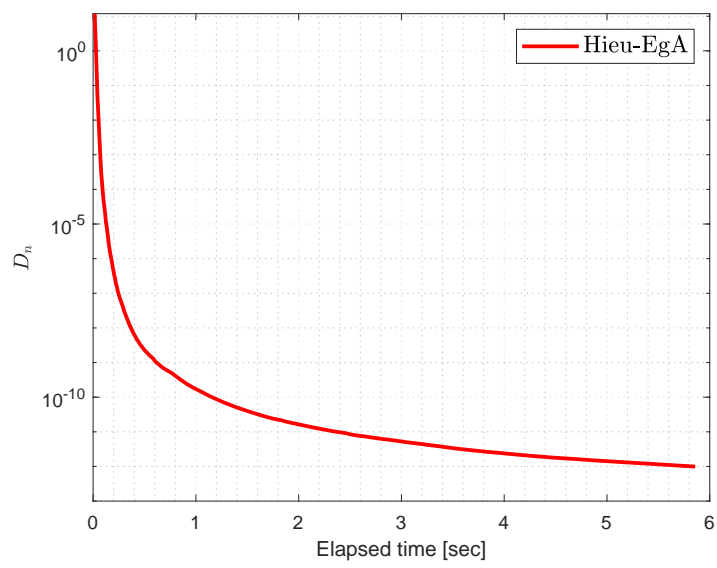

(a) CPU time in seconds

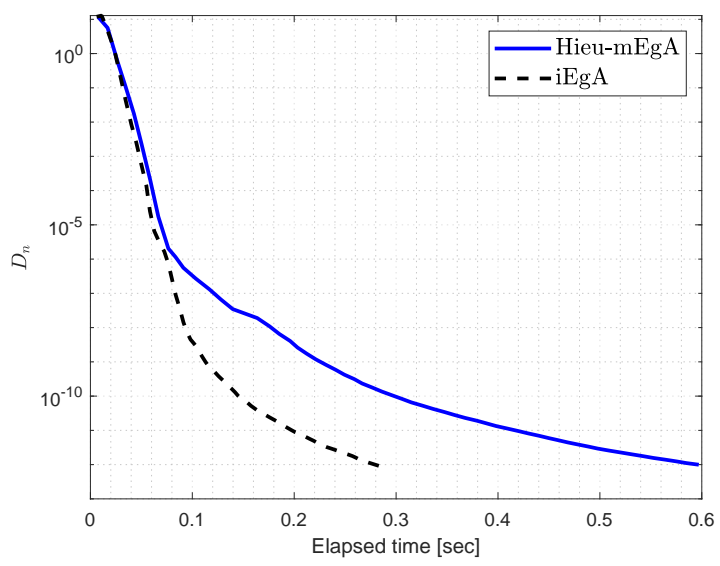

(b) CPU time in seconds

Figure 1. Example 1: Numerical comparison for Algorithm 1 while $\zeta_{n}=\frac{1}{(n+1) \log (n+3)}$.

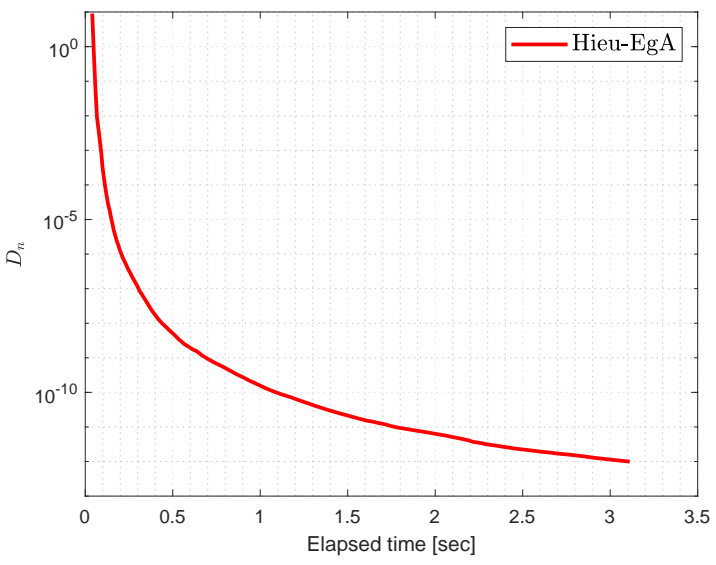

(a) CPU time in seconds

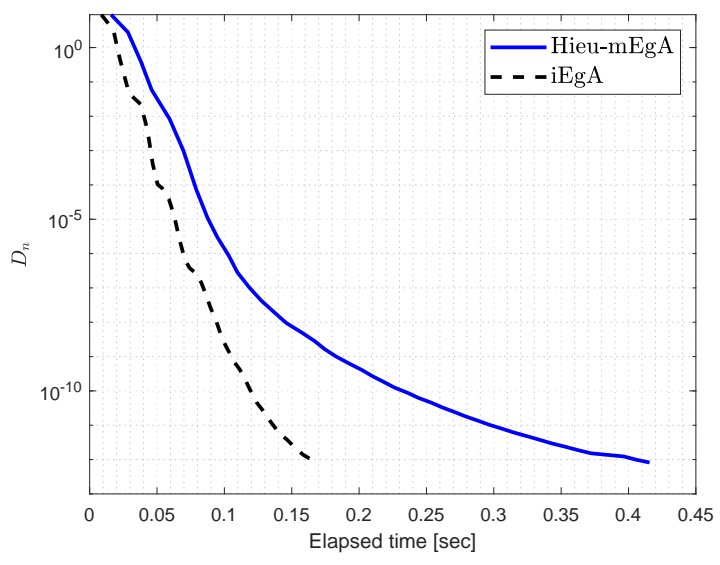

(b) CPU time in seconds

Figure 2. Example 1: Numerical comparison for Algorithm 1 while $\zeta_{n}=\frac{1}{n+1}$. 


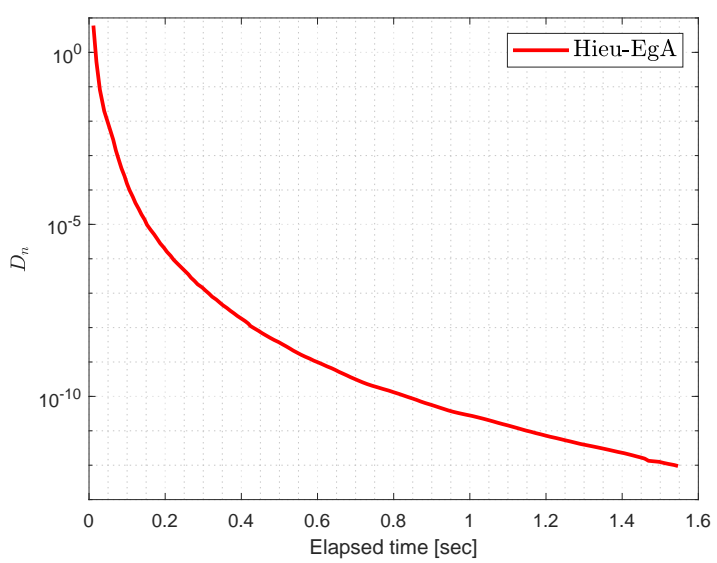

(a) CPU time in seconds

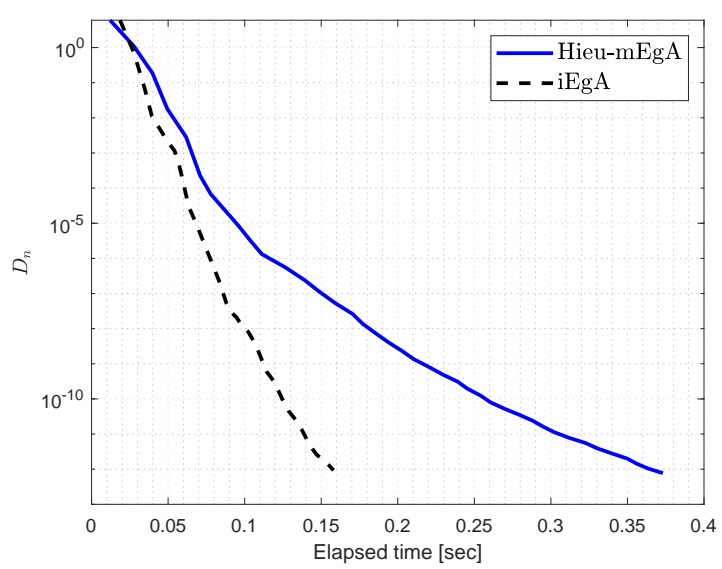

(b) CPU time in seconds

Figure 3. Example 1: Numerical comparison for Algorithm 1 while $\zeta_{n}=\frac{\log (n+3)}{n+1}$.

Example 2. Let a bifunction $f$ be defined on the convex set $\mathcal{K}$ as

$$
f(u, v)=\left\langle\left(B B^{T}+S+D\right) u, v-u\right\rangle,
$$

where $B$ is a $50 \times 50$ matrix, $S$ is a $50 \times 50$ skew-symmetric matrix, and $D$ is a $50 \times 50$ diagonal matrix. The set $\mathcal{K} \subset \mathbb{R}^{50}$ is defined by

$$
\mathcal{K}:=\left\{u \in \mathbb{R}^{50}: A u \leq b\right\}
$$

with matrix $A$ as $100 \times 50$ and vector $b$ as a non-negative vector. Observe that $f$ is monotone and Lipschitz-type constants are $c_{1}=c_{2}=\frac{\left\|B B^{T}+S+D\right\|}{2}$. We generate random matrices in our case $[B=\operatorname{rand}(n), C=\operatorname{rand}(n)$, $\left.S=0.5 C-0.5 C^{T}, D=\operatorname{diag}(\operatorname{rand}(n, 1))\right]$ and the numerical findings regarding Example 2 are shown in Figures $4-7$ with $u_{-1}=u_{0}=v_{0}=(1, \cdots, 1)$ and TOL $=10^{-12}$.

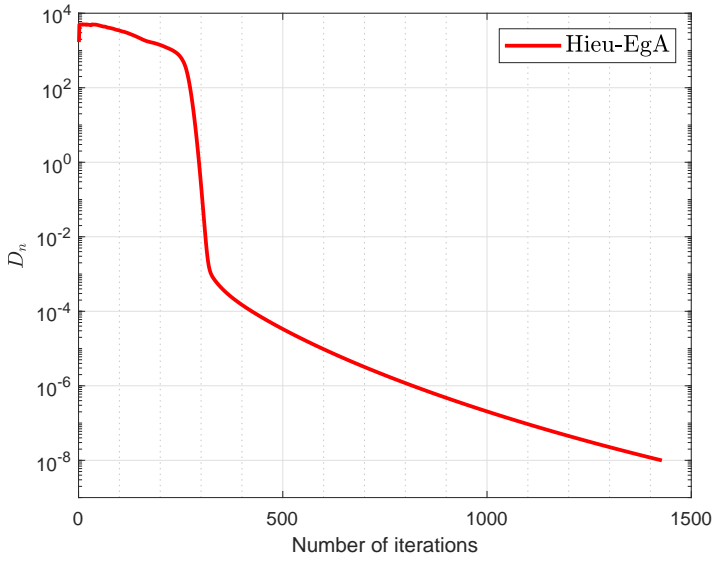

(a) Number of iterations

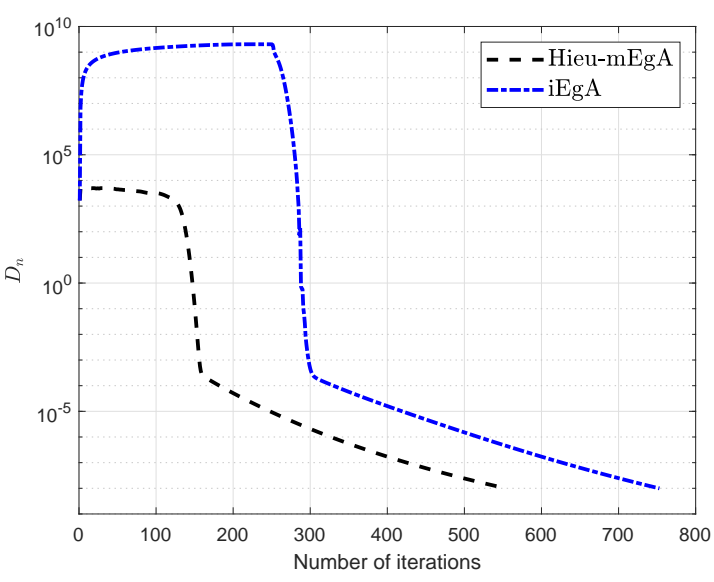

(b) Number of iterations

Figure 4. Example 2: Numerical comparison for Algorithm 1 while $\zeta_{n}=\frac{1}{n+1}$. 


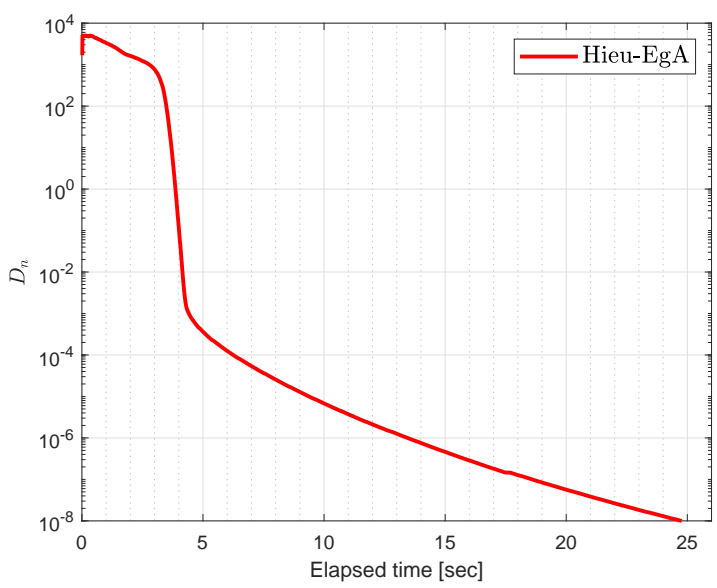

(a) CPU time in seconds

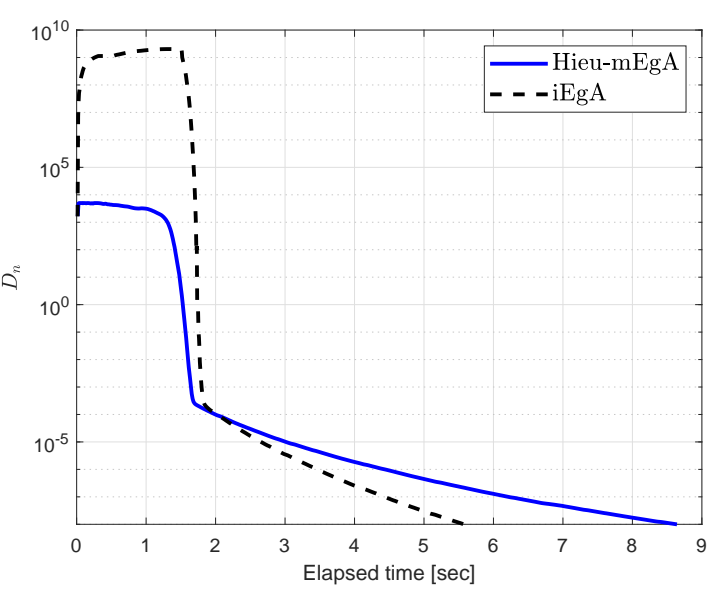

(b) CPU time in seconds

Figure 5. Example 2: Numerical comparison for Algorithm 1 while $\zeta_{n}=\frac{1}{n+1}$.

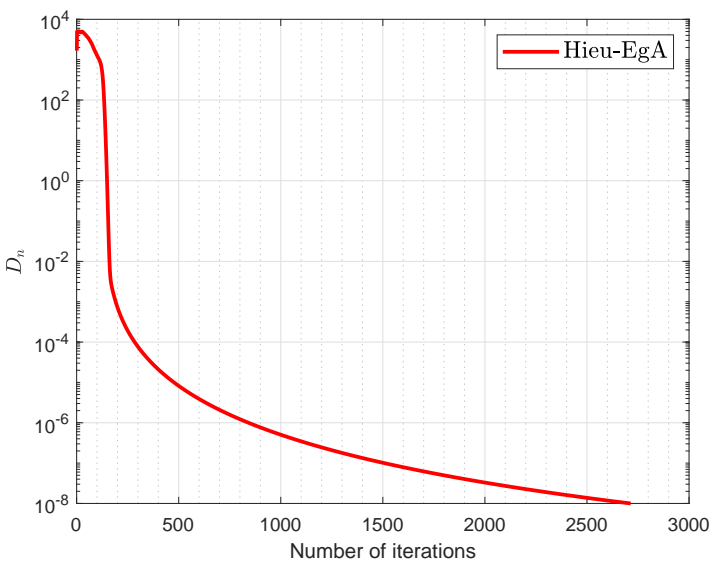

(a) Number of iterations

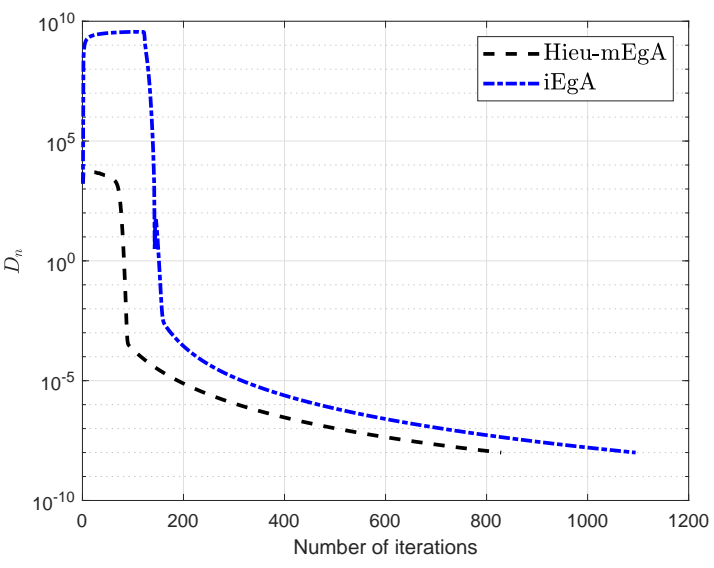

(b) Number of iterations

Figure 6. Example 2: Numerical comparison for Algorithm 1 while $\zeta_{n}=\frac{\log (n+3)}{n+1}$.

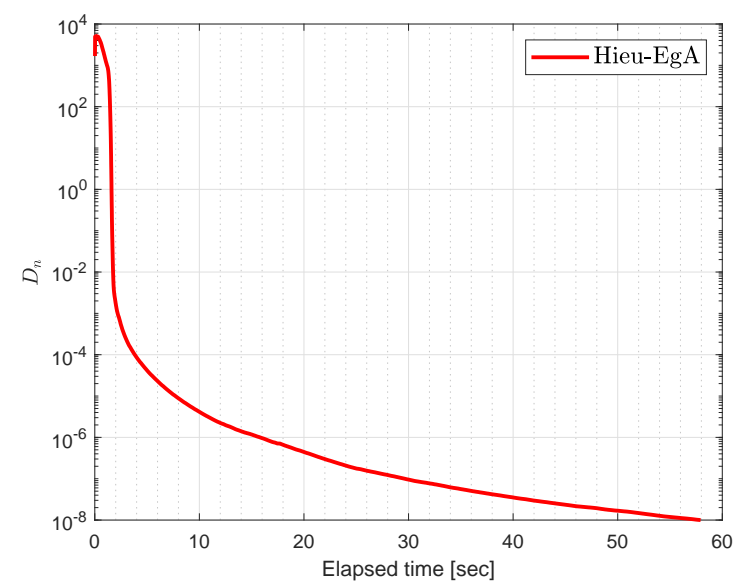

(a) CPU time in seconds

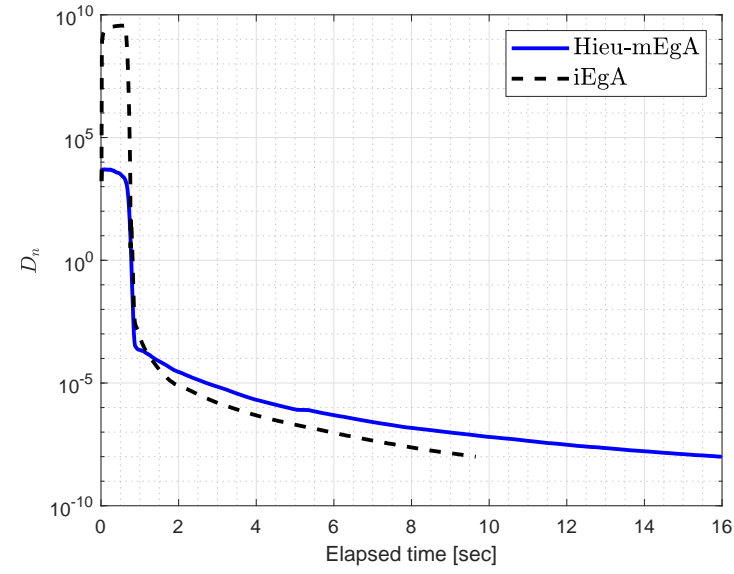

(b) CPU time in seconds

Figure 7. Example 2: Numerical comparison for Algorithm 1 while $\zeta_{n}=\frac{\log (n+3)}{n+1}$.

Example 3. Let $G: \mathbb{R}^{5} \rightarrow \mathbb{R}^{5}$ be defined by

$$
G(u)=A u+B(u)+c,
$$


where $n \times n$ symmetric semi-definite matrix $A$ and $B(u)$ is the function depends on the proximal operator [43] through $h(u)=\frac{1}{4}\|u\|^{4}$ such that

$$
B(u)=\underset{v \in \mathbb{R}^{n}}{\arg \min }\left\{\frac{\|u\|^{4}}{4}+\frac{1}{2}\|v-u\|^{2}\right\} .
$$

The feasible set $\mathcal{K}$ is considered as

$$
\mathcal{K}:=\left\{u \in \mathbb{R}^{5}:-2 \leq u_{i} \leq 5\right\} .
$$

The entries of $A$ and $c$ are taken as follows:

$$
A=\left(\begin{array}{ccccc}
3 & 1 & 0 & 1 & 2 \\
1 & 5 & -1 & 0 & 1 \\
0 & 1 & -4 & 2 & -2 \\
1 & 0 & 2 & 6 & -1 \\
2 & 1 & -2 & -1 & 4
\end{array}\right) \quad c=\left(\begin{array}{c}
1 \\
-2 \\
-1 \\
2 \\
-1
\end{array}\right)
$$

Figures 8-11 and Table 2 show the numerical results by using $u_{-1}=u_{0}=v_{0}=(1, \cdots, 1)$ and TOL $=10^{-12}$.

Table 2. Example 3: Numerical results for Figures 8-11.

\begin{tabular}{ccccccccc}
\hline & & \multicolumn{6}{c}{ Hieu-EgA [26] } & \multicolumn{2}{c}{ Hieu-mEgA [29] } & iEgA & Algorithm 1 \\
\hline $\mathbf{n}$ & TOL & $\zeta_{n}$ & Iter. & Time & Iter. & Time & Iter. & Time \\
\hline 5 & $10^{-10}$ & $\frac{1}{(n+1) \log (n+3)}$ & 440 & 29.7625 & 190 & 16.2712 & 247 & 10.8531 \\
5 & $10^{-10}$ & $\frac{1}{n+1}$ & 198 & 13.8482 & 104 & 11.8096 & 145 & 5.8483 \\
5 & $10^{-10}$ & $\frac{\log (n+3)}{n+1}$ & 178 & 12.2979 & 98 & 7.8478 & 120 & 5.2870 \\
5 & $10^{-10}$ & $\frac{1}{\sqrt{n+1}}$ & 251 & 16.7337 & 110 & 9.6097 & 148 & 6.0004 \\
\hline
\end{tabular}

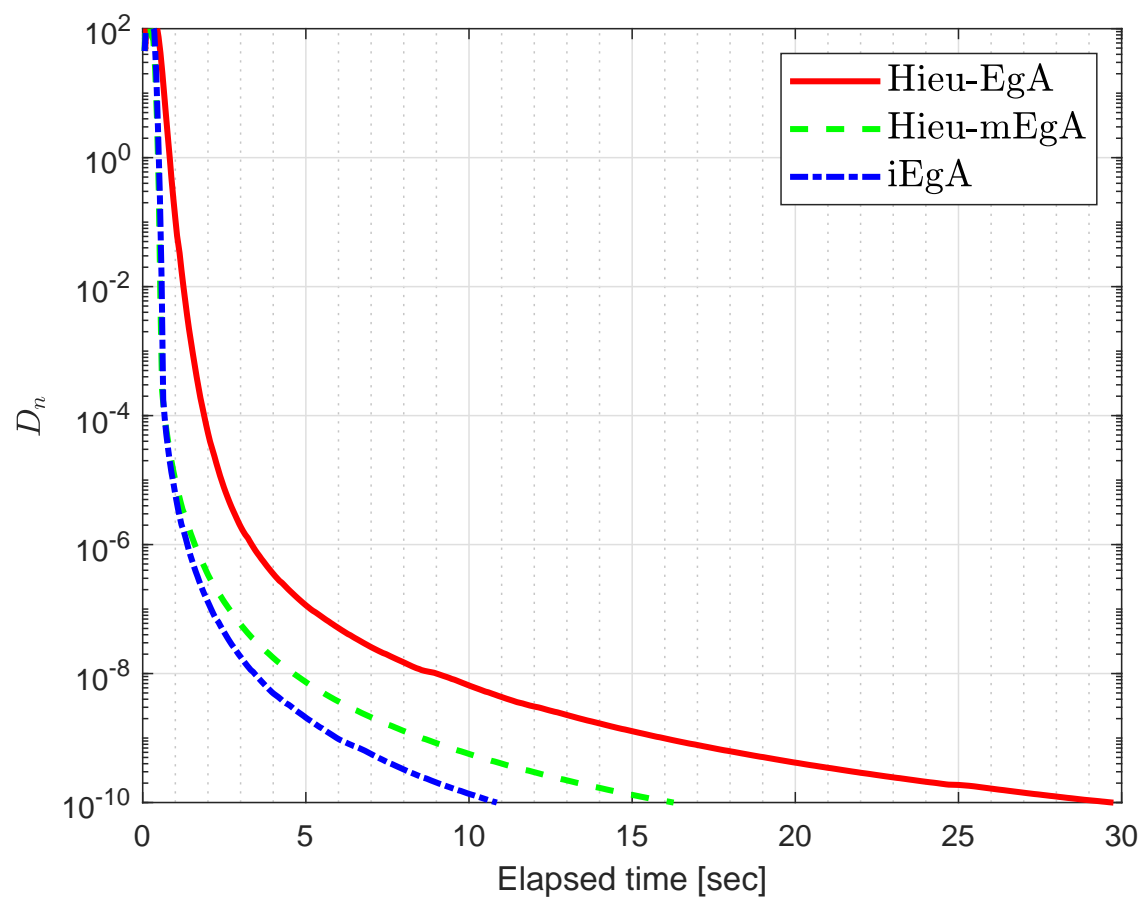

Figure 8. Example 3: Numerical comparison for Algorithm 1 while $\zeta_{n}=\frac{1}{(n+1) \log (n+3)}$. 


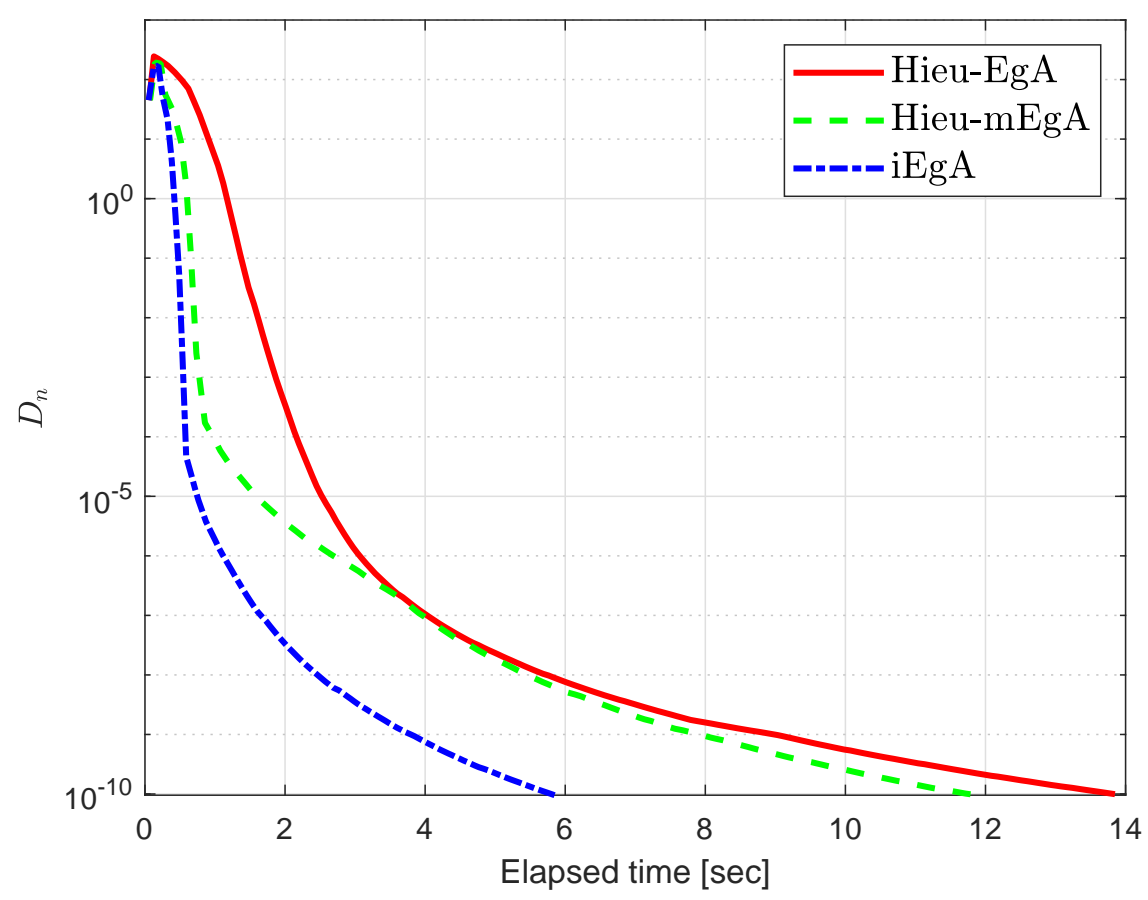

Figure 9. Example 3: Numerical comparison for Algorithm 1 while $\zeta_{n}=\frac{1}{n+1}$.

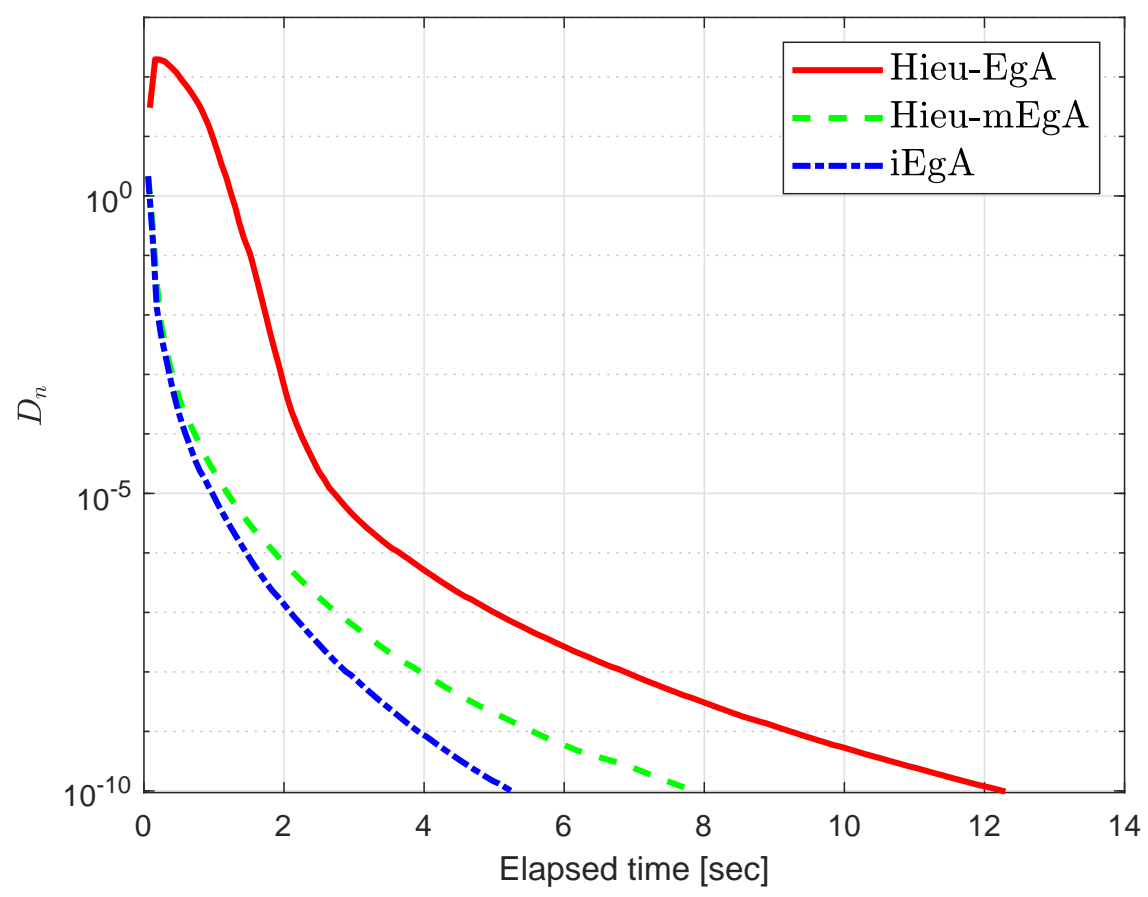

Figure 10. Example 3: Numerical comparison for Algorithm 1 while $\zeta_{n}=\frac{\log (n+3)}{n+1}$.

Example 4. Suppose that $\mathcal{K} \subset G: \mathbb{R}^{2} \rightarrow \mathbb{R}^{2}$ is defined by

$$
G\left(\begin{array}{l}
v_{1} \\
v_{2}
\end{array}\right)=\left(\begin{array}{c}
v_{1}+v_{2}+\sin \left(v_{1}\right) \\
-v_{1}+v_{2}+\sin \left(v_{2}\right)
\end{array}\right), \text { for all }\left(v_{1}, v_{2}\right) \in \mathbb{R}^{2}
$$

where $\mathcal{K}=[-5,5] \times[-5,5]$. It is easy that $G$ is Lipschitz continuous and strongly pseudomonotone operator. Figures 12-15 show the numerical results with $u_{-1}=u_{0}=v_{0}$ and $T O L=10^{-10}$. 


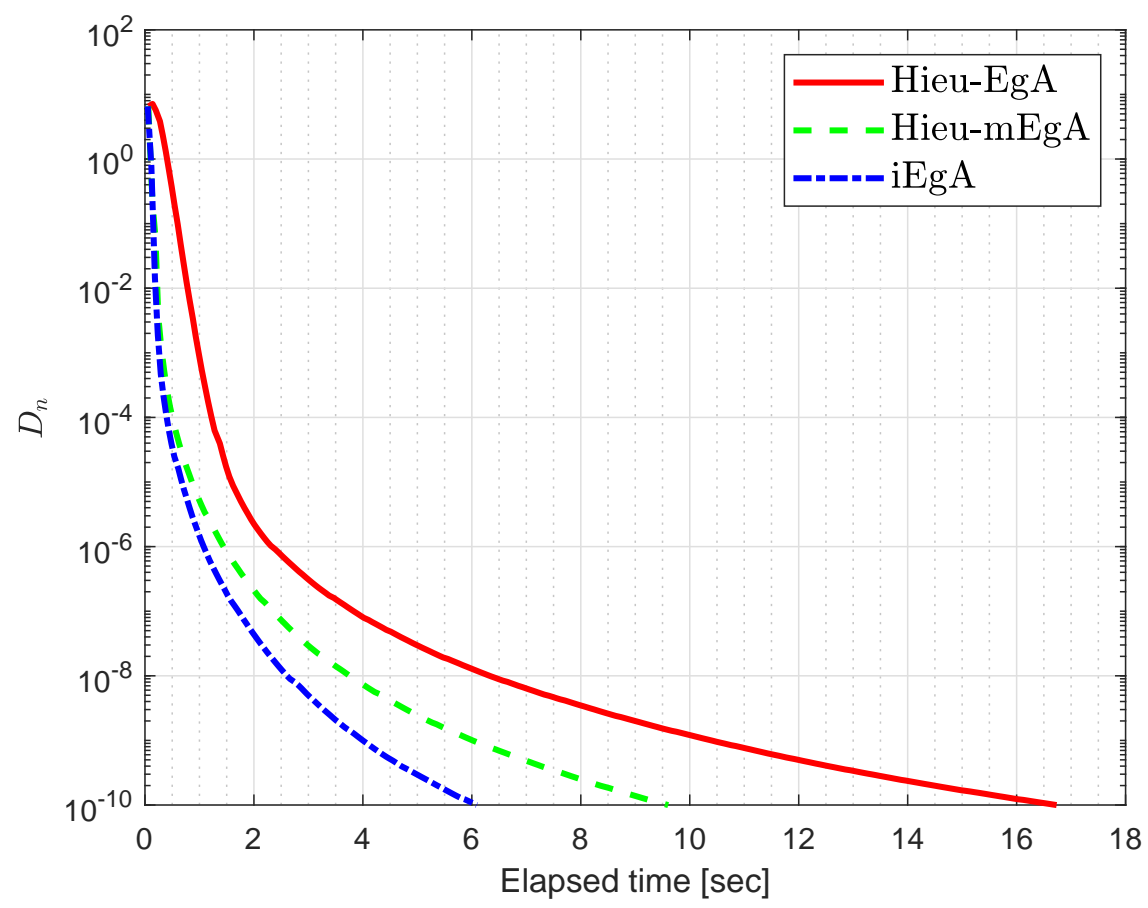

Figure 11. Example 3: Numerical comparison for Algorithm 1 while $\zeta_{n}=\frac{1}{\sqrt{n+1}}$.

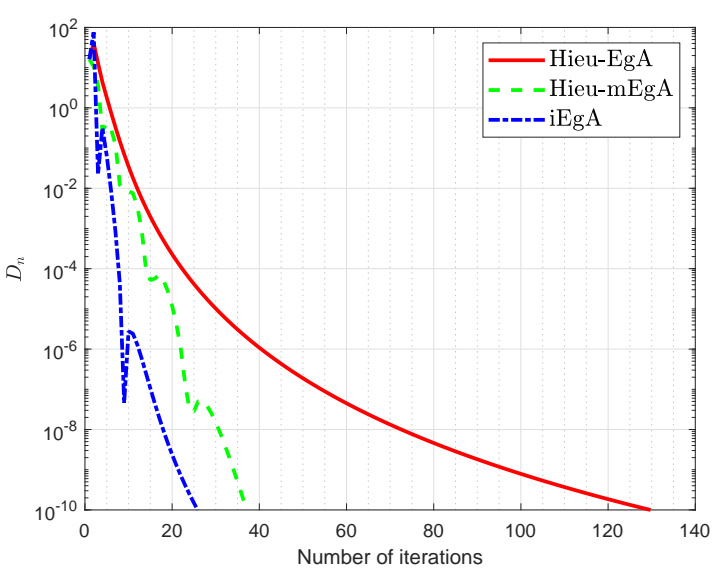

(a) Number of iterations

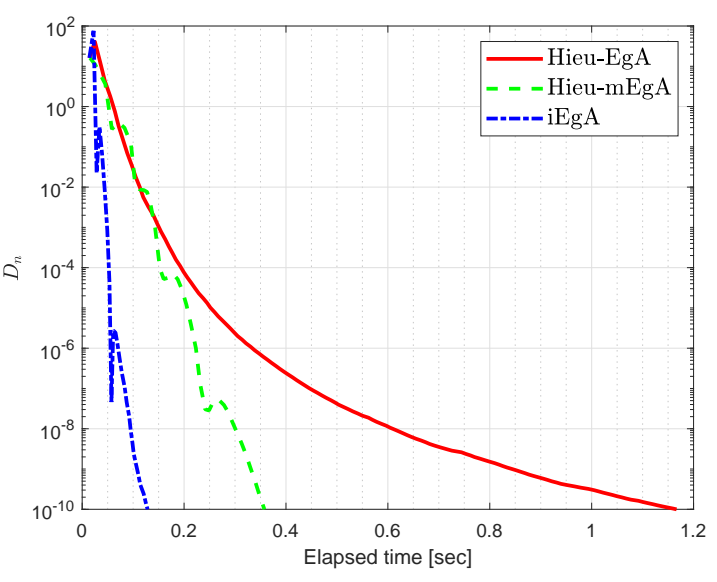

(b) CPU time in seconds

Figure 12. Example 4: Numerical comparison for Algorithm 1 while $u_{0}=(1,1)$ and $\zeta_{n}=\frac{1}{n+1}$.

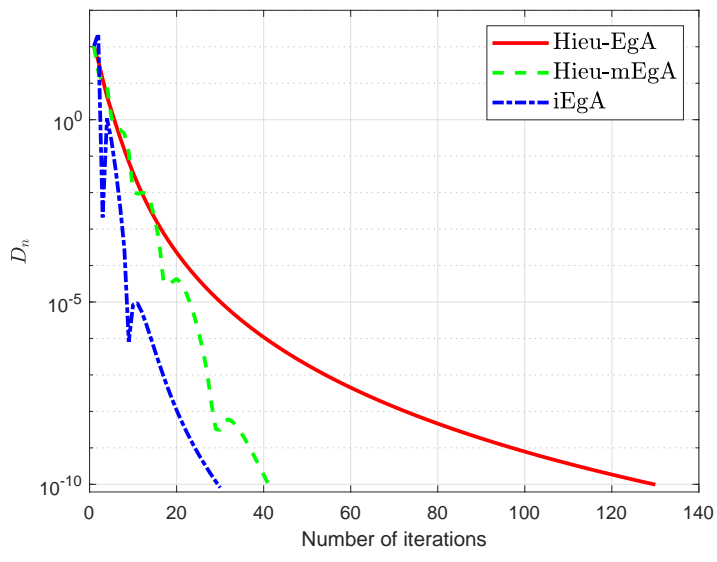

(a) Number of iterations

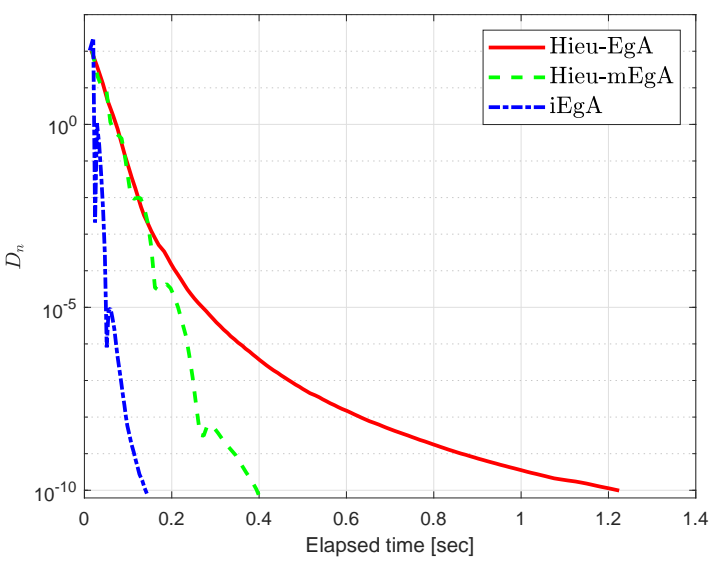

(b) CPU time in seconds

Figure 13. Example 4: Numerical comparison for Algorithm 1 while $u_{0}=(4,4)$ and $\zeta_{n}=\frac{1}{n+1}$. 


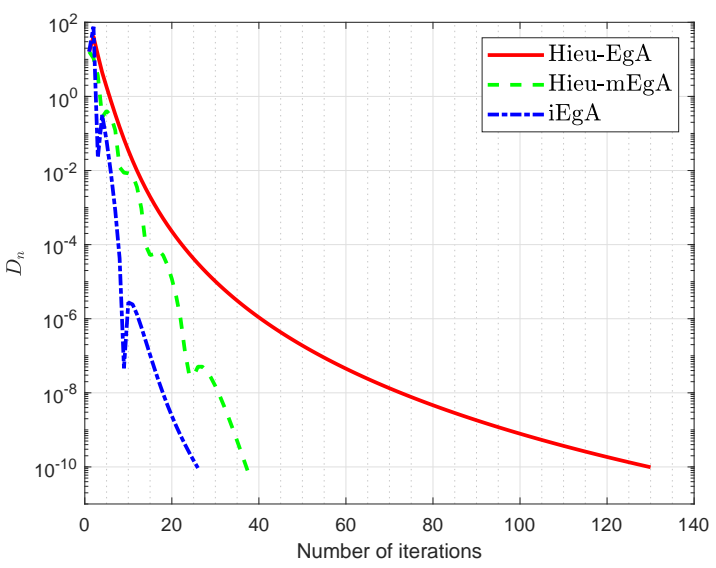

(a) Number of iterations

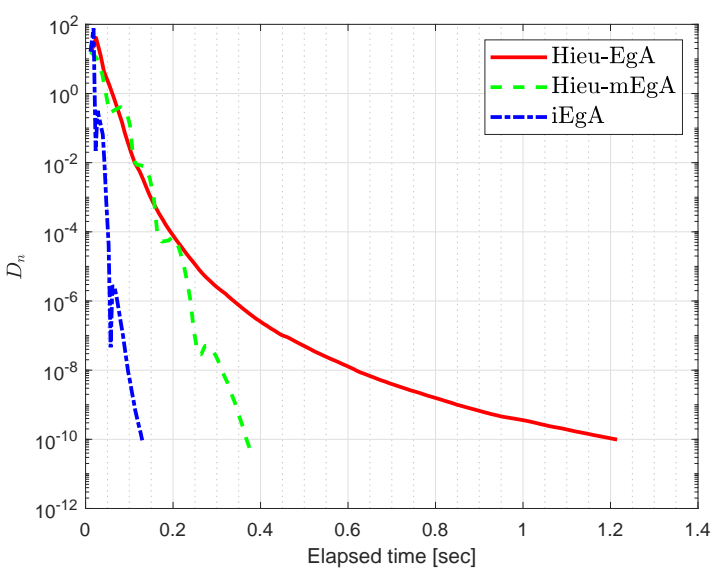

(b) CPU time in seconds

Figure 14. Example 4: Numerical comparison for Algorithm 1 while $u_{0}=(-1,-1)$ and $\zeta_{n}=\frac{1}{n+1}$.

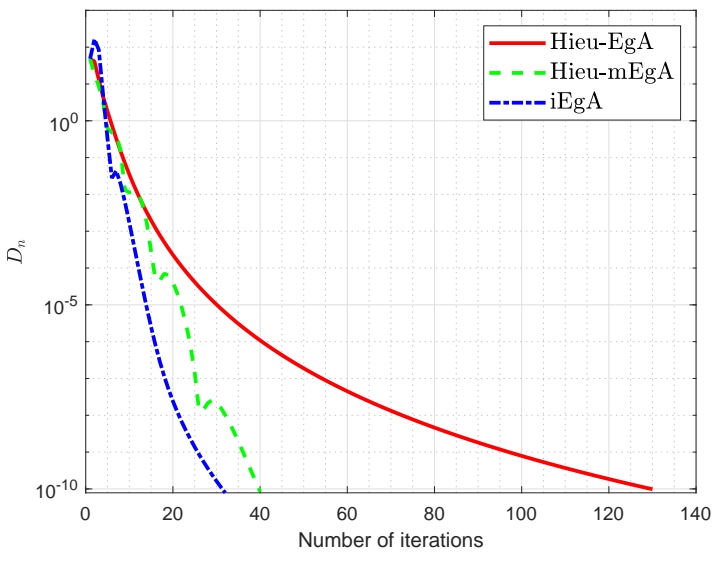

(a) Number of iterations

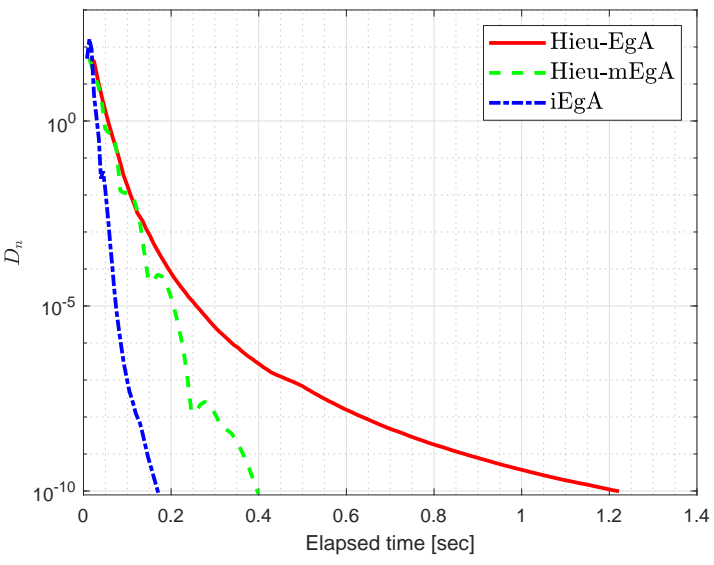

(b) CPU time in seconds

Figure 15. Example 4: Numerical comparison for Algorithm 1 while $u_{0}=(-2,-2)$ and $\zeta_{n}=\frac{1}{n+1}$.

\section{Conclusions}

In this paper, we set up a new method by combining an inertial term with an extragradient method for solving a family of strongly pseudomonotone equilibrium problems. The introduced method involves a sequence of diminishing and non-summable step size rule and the method operates without previous information of the Lipschitz-type constants. Four numerical examples are described to show the computational performance of the proposed method in relation to other existing methods. Numerical experiments clearly point out that the method with an inertial term performs better than those without an inertial term.

Author Contributions: Conceptualization, W.K. and K.M.; methodology, W.K. and K.M.; writing-original draft preparation, W.K. and K.M.; writing-review and editing, W.K. and K.M.; software, W.K. and K.M.; supervision, W.K.; and project administration and funding acquisition, K.M. All authors have read and agreed to the published version of the manuscript.

Funding: This project was supported by Rajamangala University of Technology Phra Nakhon (RMUTP).

Acknowledgments: The first author thanks the Rajamangala University of Technology Thanyaburi (RMUTT) (Grant No. NSF62D0604). The second author thanks the Rajamangala University of Technology Phra Nakhon (RMUTP).

Conflicts of Interest: The authors declare that they have no conflict of interest. 


\section{References}

1. Blum, E. From optimization and variational inequalities to equilibrium problems. Math. Stud. 1994, 63, 123-145.

2. Fan, K. A Minimax Inequality and Applications, Inequalities III; Shisha, O., Ed.; Academic Press: New York, NY, USA, 1972.

3. Bianchi, M.; Schaible, S. Generalized monotone bifunctions and equilibrium problems. J. Optim. Theory Appl. 1996, 90, 31-43. [CrossRef]

4. Bigi, G.; Castellani, M.; Pappalardo, M.; Passacantando, M. Existence and solution methods for equilibria. Eur. J. Oper. Res. 2013, 227, 1-11. [CrossRef]

5. Muu, L.; Oettli, W. Convergence of an adaptive penalty scheme for finding constrained equilibria. Nonlinear Anal. Theory Methods Appl. 1992, 18, 1159-1166. [CrossRef]

6. Combettes, P.L.; Hirstoaga, S.A.; others. Equilibrium programming in Hilbert spaces. J. Nonlinear Convex Anal. 2005, 6, 117-136.

7. Antipin, A. Equilibrium programming: Proximal methods. Comput. Math. Math. Phys. 1997, 37, $1285-1296$.

8. Giannessi, F.; Maugeri, A.; Pardalos, P.M. Equilibrium Problems: Nonsmooth Optimization and Variational Inequality Models; Springer Science \& Business Media: Berlin/Heidelberg, Germany, 2006; Volume 58.

9. Dafermos, S. Traffic Equilibrium and Variational Inequalities. Transp. Sci. 1980, 14, 42-54. [CrossRef]

10. Todorčević, V. Harmonic Quasiconformal Mappings and Hyperbolic Type Metrics; Springer International Publishing: Berlin/Heidelberg, Germany, 2019. [CrossRef]

11. ur Rehman, H.; Kumam, P.; Cho, Y.J.; Yordsorn, P. Weak convergence of explicit extragradient algorithms for solving equilibirum problems. J. Inequalities Appl. 2019, 2019. [CrossRef]

12. ur Rehman, H.; Kumam, P.; Je Cho, Y.; Suleiman, Y.I.; Kumam, W. Modified Popov's explicit iterative algorithms for solving pseudomonotone equilibrium problems. Optim. Methods Softw. 2020, 1-32. [CrossRef]

13. Todorčević, V. Subharmonic behavior and quasiconformal mappings. Anal. Math. Phys. 2019, 9, 1211-1225. [CrossRef]

14. Koskela, P.; Manojlović, V. Quasi-Nearly Subharmonic Functions and Quasiconformal Mappings. Potential Anal. 2011, 37, 187-196. [CrossRef]

15. ur Rehman, H.; Kumam, P.; Abubakar, A.B.; Cho, Y.J. The extragradient algorithm with inertial effects extended to equilibrium problems. Comput. Appl. Math. 2020, 39. [CrossRef]

16. Hammad, H.A.; ur Rehman, H.; la Sen, M.D. Advanced Algorithms and Common Solutions to Variational Inequalities. Symmetry 2020, 12, 1198. [CrossRef]

17. Hieu, D.V.; Quy, P.K.; Vy, L.V. Explicit iterative algorithms for solving equilibrium problems. Calcolo $2019,56$. [CrossRef]

18. Hieu, D.V. New inertial algorithm for a class of equilibrium problems. Numer. Algorithms 2018, 80, 1413-1436. [CrossRef]

19. Anh, P.K.; Hai, T.N. Novel self-adaptive algorithms for non-Lipschitz equilibrium problems with applications. J. Glob. Optim. 2018, 73, 637-657. [CrossRef]

20. Anh, P.N.; Anh, T.T.H.; Hien, N.D. Modified basic projection methods for a class of equilibrium problems. Numer. Algorithms 2017, 79, 139-152. [CrossRef]

21. ur Rehman, H.; Kumam, P.; Kumam, W.; Shutaywi, M.; Jirakitpuwapat, W. The Inertial Sub-Gradient Extra-Gradient Method for a Class of Pseudo-Monotone Equilibrium Problems. Symmetry 2020, 12, 463. [CrossRef]

22. ur Rehman, H.; Kumam, P.; Argyros, I.K.; Deebani, W.; Kumam, W. Inertial Extra-Gradient Method for Solving a Family of Strongly Pseudomonotone Equilibrium Problems in Real Hilbert Spaces with Application in Variational Inequality Problem. Symmetry 2020, 12, 503. [CrossRef]

23. Flåm, S.D.; Antipin, A.S. Equilibrium programming using proximal-like algorithms. Math. Program. 1996, 78, 29-41. [CrossRef]

24. Tran, D.Q.; Dung, M.L.; Nguyen, V.H. Extragradient algorithms extended to equilibrium problems. Optimization 2008, 57, 749-776. [CrossRef]

25. Korpelevich, G. The extragradient method for finding saddle points and other problems. Matecon 1976, 12, 747-756. 
26. Hieu, D.V. New extragradient method for a class of equilibrium problems in Hilbert spaces. Appl. Anal. 2017, 97, 811-824. [CrossRef]

27. Polyak, B. Some methods of speeding up the convergence of iteration methods. USSR Comput. Math. Math. Phys. 1964, 4, 1-17. [CrossRef]

28. Beck, A.; Teboulle, M. A Fast Iterative Shrinkage-Thresholding Algorithm for Linear Inverse Problems. SIAM J. Imaging Sci. 2009, 2, 183-202. [CrossRef]

29. Hieu, D.V.; Cho, Y.J.; bin Xiao, Y. Modified extragradient algorithms for solving equilibrium problems. Optimization 2018, 67, 2003-2029. [CrossRef]

30. Rehman, H.U.; Kumam, P.; Dong, Q.L.; Peng, Y.; Deebani, W. A new Popov's subgradient extragradient method for two classes of equilibrium programming in a real Hilbert space. Optimization 2020, 1-36. [CrossRef]

31. Yordsorn, P.; Kumam, P.; ur Rehman, H.; Ibrahim, A.H. A Weak Convergence Self-Adaptive Method for Solving Pseudomonotone Equilibrium Problems in a Real Hilbert Space. Mathematics 2020, 8, 1165. [CrossRef]

32. Yordsorn, P.; Kumam, P.; Rehman, H.U. Modified two-step extragradient method for solving the pseudomonotone equilibrium programming in a real Hilbert space. Carpathian J. Math. 2020, 36, 313-330.

33. Fan, J.; Liu, L.; Qin, X. A subgradient extragradient algorithm with inertial effects for solving strongly pseudomonotone variational inequalities. Optimization 2019, 1-17. [CrossRef]

34. Thong, D.V.; Hieu, D.V. Inertial extragradient algorithms for strongly pseudomonotone variational inequalities. J. Comput. Appl. Math. 2018, 341, 80-98. [CrossRef]

35. Censor, Y.; Gibali, A.; Reich, S. The Subgradient Extragradient Method for Solving Variational Inequalities in Hilbert Space. J. Optim. Theory Appl. 2010, 148, 318-335. [CrossRef] [PubMed]

36. ur Rehman, H.; Kumam, P.; Argyros, I.K.; Alreshidi, N.A.; Kumam, W.; Jirakitpuwapat, W. A Self-Adaptive Extra-Gradient Methods for a Family of Pseudomonotone Equilibrium Programming with Application in Different Classes of Variational Inequality Problems. Symmetry 2020, 12, 523. [CrossRef]

37. ur Rehman, H.; Kumam, P.; Argyros, I.K.; Shutaywi, M.; Shah, Z. Optimization Based Methods for Solving the Equilibrium Problems with Applications in Variational Inequality Problems and Solution of Nash Equilibrium Models. Mathematics 2020, 8, 822. [CrossRef]

38. ur Rehman, H.; Kumam, P.; Shutaywi, M.; Alreshidi, N.A.; Kumam, W. Inertial Optimization Based Two-Step Methods for Solving Equilibrium Problems with Applications in Variational Inequality Problems and Growth Control Equilibrium Models. Energies 2020, 13, 3292. [CrossRef]

39. Attouch, F.A.H. An Inertial Proximal Method for Maximal Monotone Operators via Discretization of a Nonlinear Oscillator with Damping. Set-Valued Var. Anal. 2001, 9, 3-11. [CrossRef]

40. Heinz, H.; Bauschke, P.L. Convex Analysis and Monotone Operator Theory in Hilbert Spaces, 2nd ed.; CMS Books in Mathematics; Springer International Publishing: Berlin/Heidelberg, Germany, 2017.

41. Ofoedu, E. Strong convergence theorem for uniformly L-Lipschitzian asymptotically pseudocontractive mapping in real Banach space. J. Math. Anal. Appl. 2006, 321, 722-728. [CrossRef]

42. Tiel, J.V. Convex Analysis: An Introductory Text, 1st ed.; Wiley: New York, NY, USA, 1984.

43. Kreyszig, E. Introductory Functional Analysis with Applications, 1st ed.; Wiley: New York, NY, USA, 1978.

Publisher's Note: MDPI stays neutral with regard to jurisdictional claims in published maps and institutional affiliations.

(C) 2020 by the authors. Licensee MDPI, Basel, Switzerland. This article is an open access article distributed under the terms and conditions of the Creative Commons Attribution (CC BY) license (http:/ / creativecommons.org/licenses/by/4.0/). 